\title{
Fast optimal load balancing algorithms for 1D partitioning is
}

\author{
Ali Pinar ${ }^{\mathrm{a}, 1}$ and Cevdet Aykanat ${ }^{\mathrm{b}, *}$ \\ ${ }^{a}$ Computational Research Division, Lawrence Berkeley National Laboratory, USA \\ ${ }^{\mathrm{b}}$ Department of Computer Engineering, Bilkent University, Ankara 06533, Turkey
}

Received 30 March 2000; revised 5 May 2004

\begin{abstract}
The one-dimensional decomposition of nonuniform workload arrays with optimal load balancing is investigated. The problem has been studied in the literature as the "chains-on-chains partitioning" problem. Despite the rich literature on exact algorithms, heuristics are still used in parallel computing community with the "hope" of good decompositions and the "myth" of exact algorithms being hard to implement and not runtime efficient. We show that exact algorithms yield significant improvements in load balance over heuristics with negligible overhead. Detailed pseudocodes of the proposed algorithms are provided for reproducibility. We start with a literature review and propose improvements and efficient implementation tips for these algorithms. We also introduce novel algorithms that are asymptotically and runtime efficient. Our experiments on sparse matrix and direct volume rendering datasets verify that balance can be significantly improved by using exact algorithms. The proposed exact algorithms are 100 times faster than a single sparse-matrix vector multiplication for 64-way decompositions on the average. We conclude that exact algorithms with proposed efficient implementations can effectively replace heuristics.
\end{abstract}

(C) 2004 Elsevier Inc. All rights reserved.

Keywords: One-dimensional partitioning; Optimal load balancing; Chains-on-chains partitioning; Dynamic programming; Iterative refinement; Parametric search; Parallel sparse matrix vector multiplication; Image-space parallel volume rendering

\section{Introduction}

This article investigates block partitioning of possibly multi-dimensional nonuniform domains over one-dimensional (1D) workload arrays. Communication and synchronization overhead is assumed to be handled implicitly by proper selection of ordering and parallel computation schemes at the beginning so that load balance is the only metric explicitly considered for decomposition. The load balancing problem in the partitioning can be modeled as the chains-on-chains partitioning $(\mathrm{CCP})$ problem with nonnegative task weights and unweighted edges between successive tasks.

\footnotetext{
This work is partially supported by The Scientific and Technical Research Council of Turkey under grant EEEAG-103E028.

*Corresponding author. Fax: +90-312-2664047.

E-mail addresses: apinar@1bl.gov (A. Pınar), aykanat@cs.bilkent. edu.tr (C. Aykanat).

${ }^{1}$ Supported by the Director, Office of Science, Division of Mathematical, Information, and Computational Sciences of the US Department of Energy under contract DE-AC03-76SF00098. One Cyclotron Road MS 50F, Berkeley, CA 94720.
}

The objective of the CCP problem is to find a sequence of $P-1$ separators to divide a chain of $N$ tasks with associated computational weights into $P$ consecutive parts so that the bottleneck value, i.e., maximum load among all processors, is minimized.

The first polynomial time algorithm for the CCP problem was proposed by Bokhari [4]. Bokhari's $O\left(N^{3} P\right)$-time algorithm is based on finding a minimum path on a layered graph. Nicol and O'Hallaron [28] reduced the complexity to $O\left(N^{2} P\right)$ by decreasing the number of edges in the layered graph. Algorithm paradigms used in following studies can be classified as dynamic programming (DP), iterative refinement, and parametric search. Anily and Federgruen [1] initiated the DP approach with an $O\left(N^{2} P\right)$-time algorithm. Hansen and Lih [13] independently proposed an $O\left(N^{2} P\right)$-time algorithm. Choi and Narahari [6], and Olstad and Manne [30] introduced asymptotically faster $O(N P)$-time, and $O((N-P) P)$-time DP-based algorithms, respectively. The iterative refinement approach starts with a partition and iteratively tries to improve the solution. The $O((N-P) P \log P)$-time 
algorithm proposed by Manne and Sørevik [23] falls into this class. The parametric-search approach relies on repeatedly probing for a partition with a bottleneck value no greater than a given value. Complexity of probing is $\theta(N)$, since each task has to be examined, but can be reduced to $O(P \log N)$ through binary search, after an initial $\theta(N)$-time prefix-sum operation on the task chain [18]. Later the complexity was reduced to $O(P \log (N / P))$ by Han et al. [12].

The parametric-search approach goes back to Iqbal's work [16] describing an $\varepsilon$-approximate algorithm which performs $O\left(\log \left(W_{\text {tot }} / \varepsilon\right)\right)$ probe calls. Here, $W_{\text {tot }}$ denotes the total task weight and $\varepsilon>0$ denotes the desired accuracy. Iqbal's algorithm exploits the observation that the bottleneck value is in the range $\left[W_{\text {tot }} / P, W_{\text {tot }}\right]$ and performs binary search in this range by making $O\left(\log \left(W_{\text {tot }} / \varepsilon\right)\right)$ probes. This work was followed by several exact algorithms involving efficient schemes for searching over bottleneck values by considering only subchain weights. Nicol and O'Hallaron $[28,29]$ proposed a search scheme that requires at most $4 N$ probes. Iqbal and Bokhari [17] relaxed the restriction of this algorithm on bounded task weight and communication cost by proposing a condensation algorithm. Iqbal [15] and Nicol [27,29] concurrently proposed an efficient search scheme that finds an optimal partition after only $O(P \log N)$ probes. Asymptotically more efficient algorithms were proposed by Frederickson [7,8] and Han et al. [12]. Frederickson proposed an $O(N)$-time optimal algorithm. Han et al. proposed a recursive algorithm with complexity $O\left(N+P^{1+\epsilon}\right)$ for any small $\epsilon>0$. These two studies have focused on asymptotic complexity, disregarding practice.

Despite these efforts, heuristics are still commonly used in the parallel computing community, and design of efficient heuristics is still an active area of research [24]. The reasons for preferring heuristics are ease of implementation, efficiency, the expectation that heuristics yield good partitions, and the misconception that exact algorithms are not affordable as a preprocessing step for efficient parallelization. By contrast, our work proposes efficient exact CCP algorithms. Implementation details and pseudocodes for proposed algorithms are presented for clarity and reproducibility. We also demonstrate that qualities of the decompositions obtained through heuristics differ substantially from those of optimal ones through experiments on a wide range of real-world problems.

For the runtime efficiency of our algorithms, we use an effective heuristic as a preprocessing step to find a good upper bound on the optimal bottleneck value. Then we exploit lower and upper bounds on the optimal bottleneck value to restrict the search space for separator-index values. This separator-index bounding scheme is exploited in a static manner in the DP algorithm, drastically reducing the number of table entries computed and referenced. A dynamic separatorindex bounding scheme is proposed for parametric search algorithms, narrowing separator-index ranges after each probe. The upper bound on the optimal bottleneck value is also exploited to find a much better initial partition for the iterative-refinement algorithm proposed by Manne and Sørevik [23]. We also propose a different iterative-refinement technique, which is very fast for small-to-medium number of processors. Observations behind this algorithm are further used to incorporate the subchain-weight concept into Iqbal's [16] approximate bisection algorithm to make it an exact algorithm.

Two applications are investigated in our experiments: sparse matrix-vector multiplication $(\mathrm{SpMxV})$ which is one of the most important kernels in scientific computing and image-space parallel volume rendering which is widely used for scientific visualization. Integer and real valued workload arrays arising in these two applications are their distinctive features. Furthermore, $\mathrm{SpMxV}$, a fine-grain application, demonstrates the feasibility of using optimal load balancing algorithms even in sparsematrix decomposition. Experiments with proposed CCP algorithms on a wide range of sparse test matrices show that 64-way decompositions can be computed 100 times faster than a single $\mathrm{SpMxV}$ time, while reducing the load imbalance by a factor of four over the most effective heuristic. Experimental results on volume rendering dataset show that exact algorithms can produce 3.8 times better 64-way decompositions than the most effective heuristic, while being only 11 percent slower on average.

The remainder of this article is organized as follows. Table 1 displays the notation used in the paper. Section 2 defines the CCP problem. A survey of existing CCP algorithms is presented in Section 3. Proposed CCP algorithms are discussed in Section 4. Load-balancing applications used in our experiments are described in Section 5 and performance results are discussed in Section 6 .

\section{Chains-on-chains partitioning (CCP) problem}

In the CCP problem, a computational problem, decomposed into a chain $\mathcal{T}=\left\langle t_{1}, t_{2}, \ldots, t_{N}\right\rangle$ of $N$ task/modules with associated positive computational weights $\mathcal{W}=\left\langle w_{1}, w_{2}, \ldots, w_{N}\right\rangle$, is to be mapped onto a chain $\mathcal{P}=\left\langle\mathcal{P}_{1}, \mathcal{P}_{2}, \ldots, \mathcal{P}_{P}\right\rangle$ of $P$ homogeneous processors. It is worth noting that there are no precedence constraints among the tasks in the chain. A subchain of $\mathcal{T}$ is defined as a subset of contiguous tasks, and the subchain consisting of tasks $\left\langle t_{i}, t_{i+1}, \ldots, t_{j}\right\rangle$ is denoted as $\mathcal{T}_{i, j}$. The computational load $W_{i, j}$ of subchain $\mathcal{T}_{i, j}$ is $W_{i, j}=\sum_{h=i}^{j} w_{h}$. From the contiguity constraint, a partition $\Pi$ should map contiguous 
Table 1

The summary of important abbreviations and symbols

\begin{tabular}{|c|c|}
\hline Notation & Explanation \\
\hline$N$ & number of tasks \\
\hline$P$ & number of processors \\
\hline $\mathcal{P}$ & processor chain \\
\hline $\mathcal{P}_{i}$ & $i$ th processor in the processor chain \\
\hline $\mathcal{T}$ & task chain, i.e., $\mathcal{T}=\left\langle t_{1}, t_{2}, \ldots, t_{N}\right\rangle$ \\
\hline$t_{i}$ & $i$ th task in the task chain \\
\hline $\mathcal{T}_{i j}$ & $\begin{array}{l}\text { subchain of tasks starting from } t_{i} \text { upto } t_{j} \text {, i.e., } \mathcal{T}_{i j}= \\
\left\langle t_{i}, t_{i+1}, \ldots, t_{j}\right\rangle\end{array}$ \\
\hline $\mathcal{T}_{i}^{p}$ & $\begin{array}{l}\text { subproblem of } p \text {-way partitioning of the first } i \text { tasks in } \\
\text { the task chain } \mathcal{T} \text {. }\end{array}$ \\
\hline$w_{i}$ & computational load of task $t_{i}$ \\
\hline$w_{\max }$ & maximum computational load among all tasks \\
\hline$w_{\text {avg }}$ & average computational load of all tasks \\
\hline$W_{i j}$ & total computational load of task subchain $T_{i j}$ \\
\hline$W_{\text {tot }}$ & total computational load \\
\hline $\mathcal{W}[i]$ & total weight of the first $i$ tasks \\
\hline$\Pi_{i}^{p}$ & $\begin{array}{l}\text { partition of first } i \text { tasks in the task chain onto the first } p \\
\text { processors in the processor chain }\end{array}$ \\
\hline$L_{p}$ & load of the $p$ th processor in a partition \\
\hline$U B$ & upperbound on the value of an optimal solution \\
\hline$L B$ & lower bound on the value of an optimal solution \\
\hline$B^{*}$ & $\begin{array}{l}\text { ideal bottleneck value, achieved when all processors } \\
\text { have equal load. }\end{array}$ \\
\hline$B_{i}^{p}$ & $\begin{array}{l}\text { optimal solution value for } p \text {-way partitioning of the } \\
\text { first } i \text { tasks }\end{array}$ \\
\hline$s_{p}$ & index of the last task assigned to the $p$ th processor. \\
\hline$S L_{p}$ & $\begin{array}{l}\text { lowest position for the } p \text { th separator index in an } \\
\text { optimal solution }\end{array}$ \\
\hline$S H_{p}$ & $\begin{array}{l}\text { highest position for the } p \text { th separator index in an } \\
\text { optimal solution }\end{array}$ \\
\hline
\end{tabular}

subchains to contiguous processors. Hence, a $P$-way chain-partition $\Pi_{N}^{P}$ of a task chain $\mathcal{T}$ with $N$ tasks onto a processor chain $\mathcal{P}$ with $P$ processors is described by a sequence $\Pi_{N}^{P}=\left\langle s_{0}, s_{1}, s_{2}, \ldots, s_{P}\right\rangle$ of $P+1$ separator indices, where $s_{0}=0 \leqslant s_{1} \leqslant \cdots \leqslant s_{P}=N$. Here, $s_{p}$ denotes the index of the last task of the $p$ th part so that $\mathcal{P}_{p}$ gets the subchain $\mathcal{T}_{s_{p-1}+1, s_{p}}$ with load $L_{p}=W_{s_{p-1}+1, s_{p}}$. The cost $C(\Pi)$ of a partition $\Pi$ is determined by the maximum processor execution time among all processors, i.e., $C(\Pi)=B=\max _{1 \leqslant p \leqslant P}\left\{L_{p}\right\}$. This $B$ value of a partition is called its bottleneck value, and the processor/ part defining it is called the bottleneck processor/part. The CCP problem can be defined as finding a mapping $\Pi_{\mathrm{opt}}$ that minimizes the bottleneck value $B_{\mathrm{opt}}=C\left(\Pi_{\mathrm{opt}}\right)$.

\section{Previous work}

Each CCP algorithm discussed in this section and Section 4 involves an initial prefix-sum operation on the task-weight array $\mathcal{W}$ to enhance the efficiency of subsequent subchain-weight computations. The prefixsum operation replaces the $i$ th entry $\mathcal{W}[i]$ with the sum of the first $i$ entries $\left(\sum_{h=1}^{i} w_{h}\right)$ so that computational load $W_{i j}$ of a subchain $\mathcal{T}_{i, j}$ can be efficiently determined as $\mathcal{W}[j]-\mathcal{W}[i-1]$ in $O(1)$-time. In our discussions, $\mathcal{W}$ is used to refer to the prefix-summed $\mathcal{W}$-array, and the $\theta(N)$ cost of this initial prefix-sum operation is considered in the complexity analysis. The presentations focus only on finding the bottleneck value $B_{\text {opt }}$, because a corresponding optimal mapping can be constructed easily by making a $\operatorname{PROBE}\left(B_{\mathrm{opt}}\right)$ call as discussed in Section 3.4.

\subsection{Heuristics}

The most commonly used partitioning heuristic is based on recursive bisection $(R B) . R B$ achieves $P$-way partitioning through $\log P$ bisection levels, where $P$ is a power of 2. At each bisection step in a level, the current chain is divided evenly into two subchains. Although optimal division can be easily achieved at every bisection step, the sequence of optimal bisections may lead to poor load balancing. $R B$ can be efficiently implemented in $O(N+P \log N)$ time by first performing a prefix-sum operation on the workload array $\mathcal{W}$, with complexity $O(N)$, and then making $P-1$ binary searches in the prefix-summed $\mathcal{W}$-array, each with complexity $O(\log N)$.

Miguet and Pierson [24] proposed two other heuristics. The first heuristic $(H 1)$ computes the separator values such that $s_{p}$ is the largest index such that $W_{1, s_{p}} \leqslant p B^{*}$, where $B^{*}=W_{\text {tot }} / P$ is the ideal bottleneck value, and $W_{\text {tot }}=\sum_{i=1}^{N} w_{i}$ denotes sum of all task weights. The second heuristic $(H 2)$ further refines the separator indices by incrementing each $s_{p}$ value found in $H 1$ if $\left(W_{1, s_{p+1}}-p B^{*}\right)<\left(p B^{*}-W_{1, s_{p}}\right)$. These two heuristics can also be implemented in $O(N+P \log N)$ time by performing $P-1$ binary searches in the prefixsummed $\mathcal{W}$-array. Miguet and Pierson [24] have already proved the upper bounds on the bottleneck values of the partitions found by $H 1$ and $H 2$ as $B_{H 1}, B_{H 2}<B^{*}+$ $w_{\max }$, where $w_{\max }=\max _{1 \leqslant p \leqslant N}\left\{w_{i}\right\}$ denotes the maximum task weight. The following lemma establishes a similar bound for the $R B$ heuristic.

Lemma 3.1. Let $\Pi_{R B}=\left\langle s_{0}, s_{1}, \ldots, s_{P}\right\rangle$ be an $R B$ solution to a CCP problem $(\mathcal{W}, N, P)$. Then $B_{R B}=C\left(\Pi_{R B}\right)$ satisfies $B_{R B} \leqslant B^{*}+w_{\max }(P-1) / P$.

Proof. Consider the first bisection step. There exists a pivot index $1 \leqslant i_{1} \leqslant N$ such that both sides weigh less than $W_{\text {tot }} / 2$ without the $i_{1}$ th task, and more than $W_{\text {tot }} / 2$ with it. That is,

$W_{1, i_{1}-1}, W_{i_{1}+1, N} \leqslant W_{\text {tot }} / 2 \leqslant W_{1, i_{1}}, W_{i_{1}, N}$.

The worst case for $R B$ occurs when $w_{i_{1}}=w_{\max }$ and $W_{1, i_{1}-1}=W_{i_{1}+1, N}=\left(W_{\text {tot }}-w_{\max }\right) / 2$. Without loss of generality, assume that $t_{i_{1}}$ is assigned to the left part so that $s_{P / 2}=i_{1}$ and $W_{1, s_{P / 2}}=W_{\text {tot }} / 2+w_{\max } / 2$. In a similar worst-case bisection of $\mathcal{T}_{1, s_{P / 2}}$, there exists an 
index $i_{2}$ such that $w_{i_{2}}=w_{\max }$ and $W_{1, i_{2}-1}=W_{i_{2}+1, s_{P / 2}}=$ $\left(W_{\text {tot }}-w_{\max }\right) / 4$, and $t_{i_{2}}$ is assigned to the left part so that $s_{P / 4}=i_{2}$ and $W_{1, s_{P / 4}}=\left(W_{\text {tot }}-w_{\max }\right) / 4+w_{\max }=$ $W_{\text {tot }} / 4+(3 / 4) w_{\max }$. For a sequence of $\log P$ such worstcase bisection steps on the left parts, processor $\mathcal{P}_{1}$ will be the bottleneck processor with load $B_{R B}=W_{1, s_{1}}=$ $W_{\text {tot }} / P+w_{\max }(P-1) / P$.

\subsection{Dynamic programming}

The overlapping subproblem space can be defined as $\mathcal{T}_{i}^{p}$, for $p=1,2, \ldots, P$ and $i=p, p+1, \ldots, N-P+p$, where $\mathcal{T}_{i}^{p}$ denotes a $p$-way CCP of prefix task-subchain $\mathcal{T}_{1, i}=\left\langle t_{1}, t_{2}, \ldots, t_{i}\right\rangle$ onto prefix processor-subchain $\mathcal{P}_{1, p}=\left\langle\mathcal{P}_{1}, \mathcal{P}_{2}, \ldots, \mathcal{P}_{p}\right\rangle$. Notice that index $i$ is restricted to $p \leqslant i \leqslant N-P+p$ range because there is no merit in leaving a processor empty. From this subproblem space definition, the optimal substructure property of the CCP problem can be shown by considering an optimal mapping $\Pi_{i}^{p}=\left\langle s_{0}, s_{1}, \ldots, s_{p}=i\right\rangle$ with a bottleneck value $B_{i}^{p}$ for the CCP subproblem $\mathcal{T}_{i}^{p}$. If the last processor is not the bottleneck processor in $\Pi_{i}^{p}$, then $\Pi_{s_{p-1}}^{p-1}=\left\langle s_{0}, s_{1}, \ldots, s_{p-1}\right\rangle$ should be an optimal mapping for the subproblem $\mathcal{T}_{s_{p-1}}^{p-1}$. Hence, recursive definition for the bottleneck value of an optimal mapping is

$B_{i}^{p}=\min _{p-1 \leqslant j<i}\left\{\max \left\{B_{j}^{p-1}, W_{j+1, i}\right\}\right\}$.

In (1), searching for index $j$ corresponds to searching for separator $s_{p-1}$ so that the remaining subchain $\mathcal{T}_{j+1, i}$ is assigned to the last processor $\mathcal{P}_{p}$ in an optimal mapping $\Pi_{i}^{p}$ of $\mathcal{T}_{i}^{p}$. The bottleneck value $B_{N}^{P}$ of an optimal mapping can be computed using (1) in a bottom-up fashion starting from $B_{i}^{1}=W_{1, i}$ for $i=1,2, \ldots, N$. An initial prefix-sum on the workload array $\mathcal{W}$ enables constant-time computation of subchain weight of the form $W_{j+1, i}$ through $W_{j+1, i}=\mathcal{W}[i]-\mathcal{W}[j]$. Computing $B_{i}^{p}$ using (1) takes $O(N-p)$ time for each $i$ and $p$, and thus the algorithm takes $O\left((N-P)^{2} P\right)$ time since the number of distinct subproblems is equal to $(N-P+1) P$.

Choi and Narahari [6], and Olstad and Manne [30] reduced the complexity of this scheme to $O(N P)$ and $O((N-P) P)$, respectively, by exploiting the following observations that hold for positive task weights. For a fixed $p$ in (1), the minimum index value $j_{i}^{p}$ defining $B_{i}^{p}$ cannot occur at a value less than the minimum index value $j_{i-1}^{p}$ defining $B_{i-1}^{p}$, i.e., $j_{i-1}^{p} \leqslant j_{i}^{p} \leqslant(i-1)$. Hence, the search for the optimal $j_{i}^{p}$ can start from $j_{i-1}^{p}$. In (1), $B_{j}^{p-1}$ for a fixed $p$ is a nondecreasing function of $j$, and $W_{j+1, i}$ for a fixed $i$ is a decreasing function of $j$, reducing to 0 at $j=i$. Thus, two cases occur in a semi-closed interval $\left[j_{i-1}^{p}, i\right)$ for $j$. If $W_{j+1, i}>B_{j}^{p-1}$ initially, then these two functions intersect in $\left[j_{i-1}^{p}, i\right)$. In this case, the search for $j_{i}^{p}$ continues until $W_{j^{*}+1, i} \leqslant B_{j}^{p-1}$ and then only $j^{*}$ and $j^{*}-1$ are considered for setting $j_{i}^{p}$ with $j_{i}^{p}=j^{*}$ if
$B_{j^{*}}^{p-1} \leqslant W_{j, i}$ and $j_{i}^{p}=j^{*}-1$ otherwise. Note that this scheme automatically detects $j_{i}^{p}=i-1$ if $W_{j+1, i}$ and $B_{j}^{p-1}$ intersect in the open interval $(i-1, i)$. However if, $W_{j+1, i} \leqslant B_{j}^{p-1}$ initially, then $B_{j}^{p-1}$ lies above $W_{j+1, i}$ in the closed interval $\left[j_{i-1}^{p}, i\right]$. In this case, the minimum value occurs at the first value of $j$, i.e., $j_{i}^{p}=j_{i-1}^{p}$. These improvements lead to an $O((N-P) P)$-time algorithm since computation of all $B_{i}^{p}$ values for a fixed $p$ makes $O(N-P)$ references to already computed $B_{j}^{p-1}$ values. Fig. 1 displays a run-time efficient implementation of this $O((N-P) P)$-time DP algorithm which avoids the explicit min-max operation required in (1). In Fig. $1, B_{i}^{p}$ values are stored in a table whose entries are computed in row-major order.

\subsection{Iterative refinement}

The algorithm proposed by Manne and Sørevik [23], referred to here as the MS algorithm, finds a sequence of nonoptimal partitions such that there is only one way each partition can be improved. For this purpose, they introduce the leftist partition $(L P)$. Consider a partition $\Pi$ such that $\mathcal{P}_{p}$ is the leftmost processor containing at least two tasks. $\Pi$ is defined as an LP if increasing the load of any processor $\mathcal{P}_{\ell}$ that lies to the right of $\mathcal{P}_{p}$ by augmenting the last task of $\mathcal{P}_{\ell-1}$ to $\mathcal{P}_{\ell}$ makes $\mathcal{P}_{\ell}$ a bottleneck processor with a load $\geqslant C(\Pi)$. Let $\Pi$ be an LP with bottleneck processor $\mathcal{P}_{b}$ and bottleneck value $B$. If $\mathcal{P}_{b}$ contains only one task, then $\Pi$ is optimal. On the other hand, assume that $\mathcal{P}_{b}$ contains at least two tasks. The refinement step, which is shown by the inner whileloop in Fig. 2, tries to find a new LP of lower cost by successively removing the first task of $\mathcal{P}_{p}$ and augmenting it to $\mathcal{P}_{p-1}$ for $p=b, b-1, \ldots$, until $L_{p}<B$. Refinement fails when the while-loop proceeds until $p=1$ with $L_{p} \geqslant B$. Manne and Sørevik proved that a successful refinement of an LP gives a new LP and the LP is optimal if the refinement fails. They proposed using an initial LP in which the $P-1$ leftmost processors each

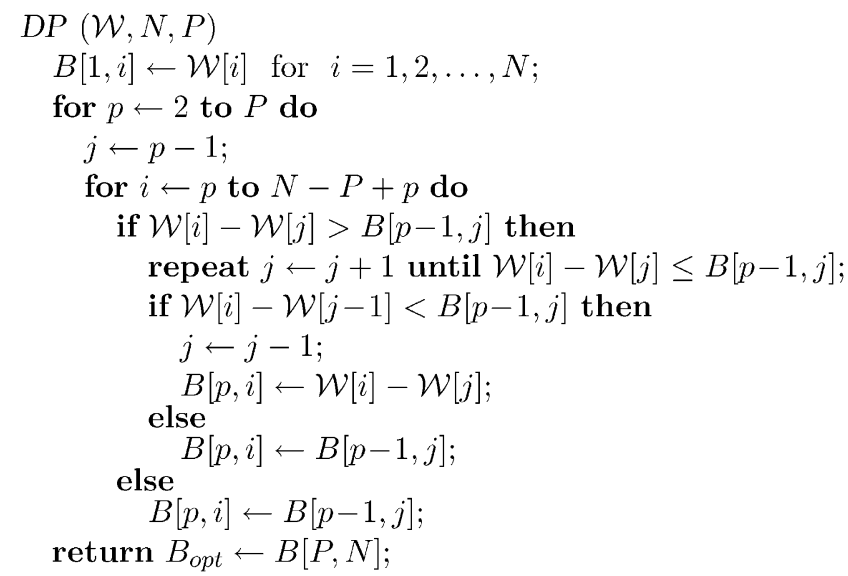

Fig. 1. $O((N-P) P)$-time dynamic-programming algorithm proposed by Choi and Narahari [6], and Olstad and Manne [30]. 
has only one task and the last processor contains the remaining tasks. The MS algorithm moves each separator index at most $N-P$ times so that the total number of separator-index moves is $O(P(N-P))$. A max-heap is maintained for the processor loads to find a bottleneck processor at the beginning of each repeatloop iteration. The cost of each separator-index move is $O(\log P)$ since it necessitates one decrease-key and one increase-key operations. Thus the complexity of the MS algorithm is $O(P(N-P) \log P)$.

\subsection{Parametric search}

The parametric-search approach relies on repeated probing for a partition $\Pi$ with a bottleneck value no

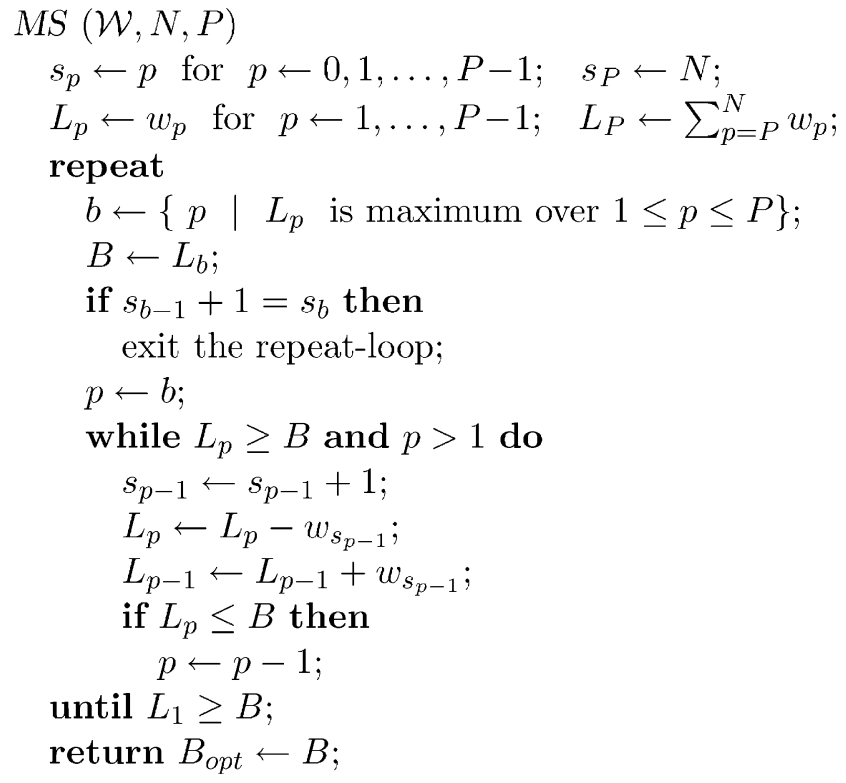

Fig. 2. Iterative refinement algorithm proposed by Manne and Sørevik [23]. greater than a given $B$ value. Probe algorithms exploit the greedy-choice property for existence and construction of $\Pi$. The greedy choice here is to minimize remaining work after loading processor $\mathcal{P}_{p}$ subject to $L_{p} \leqslant B$ for $p=1, \ldots, P-1$ in order. $P R O B E(B)$ functions given in Fig. 3 exploit this greedy property as follows. $P R O B E$ finds the largest index $s_{1}$ so that $W_{1, s_{1}} \leqslant B$, and assigns subchain $\mathcal{T}_{1, s_{1}}$ to processor $\mathcal{P}_{1}$ with load $L_{1}=W_{1, s_{1}}$. Hence, the first task in the second processor is $t_{s_{1}+1}$. PROBE then similarly finds the largest index $s_{2}$ so that $W_{s_{1}+1, s_{2}} \leqslant B$, and assigns the subchain $\mathcal{T}_{s_{1}+1, s_{2}}$ to processor $\mathcal{P}_{2}$. This process continues until either all tasks are assigned or all processors are exhausted. The former and latter cases indicate feasibility and infeasibility of $B$, respectively.

Fig. 3(a) illustrates the standard probe algorithm. The indices $s_{1}, s_{2}, \ldots, s_{P-1}$ are efficiently found through binary search $(B I N S R C H)$ on the prefix-summed $\mathcal{W}$ array. In this figure, $B I N S R C H(\mathcal{W}, i, N, B s u m)$ searches $\mathcal{W}$ in the index range $[i, N]$ to compute the index $i \leqslant j \leqslant N$ such that $\mathcal{W}[j] \leqslant$ Bsum and $\mathcal{W}[j+1]>$ Bsum. The complexity of the standard probe algorithm is $O(P \log N)$. Han et al. [12] proposed an $O(P \log N / P)$ time probe algorithm (see Fig. 3(b)) exploiting $P$ repeated binary searches on the same $\mathcal{W}$ array with increasing search values. Their algorithm divides the chain into $P$ subchains of equal length. At each probe, a linear search is performed on the weights of the last tasks of these $P$ subchains to find out in which subchain the search value could be, and then binary search is performed on the respective subchain of length $N / P$. Note that since the probe search values always increase, linear search can be performed incrementally, that is, search continues from the last subchain that was searched to the right with $O(P)$ total cost. This gives a total cost of $O(P \log (N / P))$ for $P$ binary searches thus for the probe function.

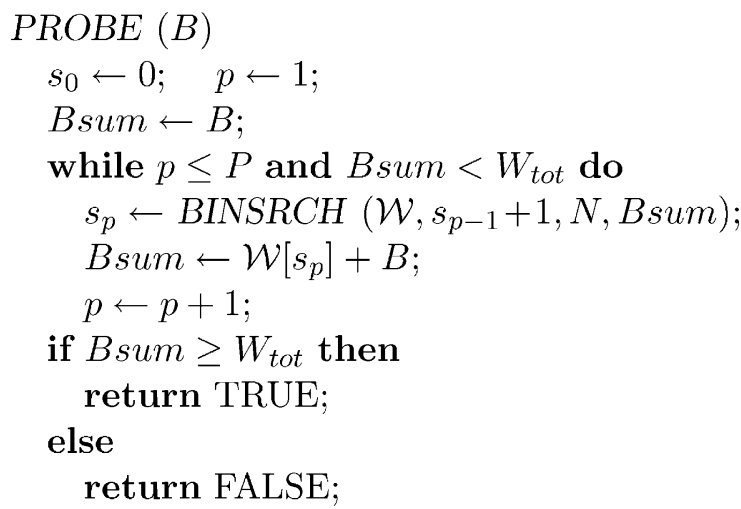

(a)

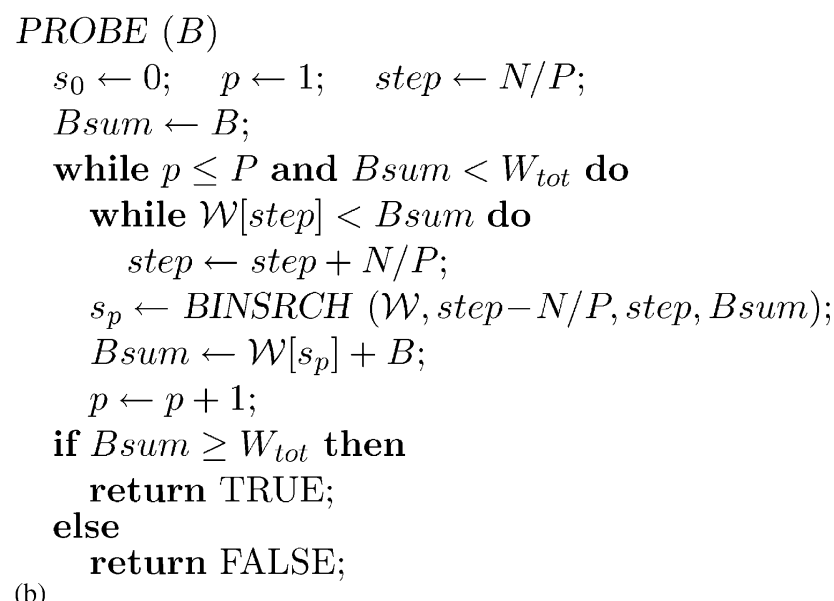

(b)

Fig. 3. (a) Standard probe algorithm with $O(P \log N)$ complexity, (b) $O(P \log (N / P))$-time probe algorithm proposed by Han et al. [12]. 


\subsubsection{Bisection as an approximation algorithm}

Let $f(B)$ be the binary-valued function where $f(B)=$ 1 if $\operatorname{PROBE}(B)$ is true and $f(B)=0$ if $\operatorname{PROBE}(B)$ is false. Clearly, $f(B)$ is nondecreasing in $B$, and $B_{\text {opt }}$ lies between $L B=B^{*}=W_{\text {tot }} / P$ and $U B=W_{\text {tot }}$. These observations are exploited in the bisection algorithm leading to an efficient $\varepsilon$-approximate algorithm, where $\varepsilon$ is the desired precision. The interval $\left[W_{\text {tot }} / P, W_{\text {tot }}\right]$ is conceptually discretized into $\left(W_{\text {tot }}-W_{\text {tot }} / P\right) / \varepsilon$ bottleneck values, and binary search is used in this range to find the minimum feasible bottleneck value $B_{\text {opt }}$. The bisection algorithm, as illustrated in Fig. 4, performs $O\left(\log \left(W_{\text {tot }} / \varepsilon\right)\right)$ PROBE calls, and each $P R O B E$ call costs $O(P \log (N / P))$. Hence, the bisection algorithm runs in $O\left(N+P \log (N / P) \log \left(W_{\text {tot }} / \varepsilon\right)\right)$ time, where $O(N)$ cost comes from the initial prefix-sum operation on $\mathcal{W}$. The performance of this algorithm deteriorates when $\log \left(W_{\text {tot }} / \varepsilon\right)$ is comparable with $N$.

\subsubsection{Nicol's algorithm}

Nicol's algorithm [27] exploits the fact that any candidate $B$ value is equal to weight $W_{i, j}$ of a subchain. A naive solution is to generate all subchain weights of the form $W_{i, j}$, sort them, and then use binary search to find the minimum $W_{a, b}$ value for which $\operatorname{PROBE}\left(W_{a, b}\right)=$ TRUE. Nicol's algorithm efficiently searches for the earliest range $W_{a, b}$ for which $B_{\text {opt }}=W_{a, b}$ by considering each processor in order as a candidate bottleneck processor in an optimal mapping. Let $\Pi_{\mathrm{opt}}$ be the optimal mapping constructed by greedy $P R O B E\left(B_{\text {opt }}\right)$, and let processor $\mathcal{P}_{b}$ be the first bottleneck processor with load $B_{\text {opt }}$ in $\Pi_{\text {opt }}=\left\langle s_{0}, s_{1}, \ldots, s_{b}, \ldots, s_{P}\right\rangle$. Under these assumptions, this greedy construction of $\Pi_{\mathrm{opt}}$ ensures that each processor $\mathcal{P}_{p}$ preceding $\mathcal{P}_{b}$ is loaded as much as possible with $L_{p}<B_{\text {opt }}$, for $p=1,2, \ldots, b-1$ in $\Pi_{\text {opt }}$. Here, $\operatorname{PROBE}\left(L_{p}\right)=$ FALSE since $L_{p}<B_{\text {opt }}$, and $\operatorname{PROBE}\left(L_{p}+W_{s_{p}+1}\right)=$ TRUE since adding one more task to processor $\mathcal{P}_{p}$ increases its load to $L_{p}+$ $w_{s_{p}+1}>B_{\text {opt }}$. Hence, if $b=1$ then $s_{1}$ is equal to the smallest index $i_{1}$ such that $\operatorname{PROBE}\left(W_{1, i_{1}}\right)=$ TRUE, and

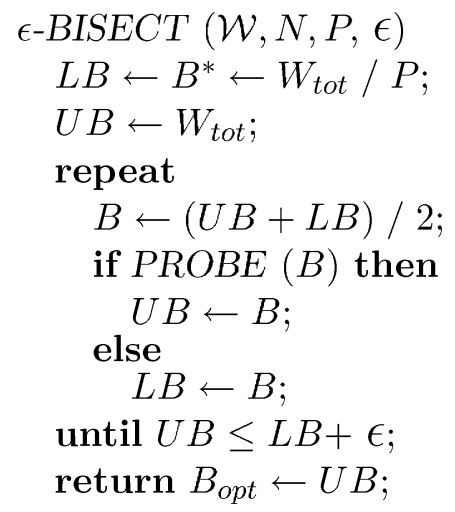

Fig. 4. Bisection as an $\varepsilon$-approximation algorithm.
$B_{\text {opt }}=B_{1}=W_{1, s_{1}}$. However, if $b>1$, then because of the greedy choice property $\mathcal{P}_{1}$ should be loaded as much as possible without exceeding $B_{\text {opt }}=B_{b}<B_{1}$, which implies that $s_{1}=i_{1}-1$, and hence $L_{1}=W_{1, i_{1}-1}$. If $b=2$, then $s_{2}$ is equal to the smallest index $i_{2}$ such that $\operatorname{PROBE}\left(W_{i_{1}, i_{2}}\right)=$ TRUE, and $B_{\mathrm{opt}}=B_{2}=W_{i_{1}, i_{2}}$. If $b>2$, then $s_{2}=i_{2}-1$. We iterate for $b=1,2, \ldots, P-$ 1 , computing $i_{b}$ as the smallest index for which $\operatorname{PROBE}\left(W_{i_{b-1}, i_{b}}\right)=$ TRUE and save $B_{b}=W_{i_{b-1}, i_{b}}$ with $i_{P}=N$. Finally, the optimal bottleneck value is selected as $B_{\text {opt }}=\min _{1 \leqslant b \leqslant P} B_{b}$.

Fig. 5 illustrates Nicol's algorithm. As seen in this figure, given $i_{b-1}, i_{b}$ is found by performing a binary search over all subchain weights of the form $W_{i_{b-1}, j}$, for $i_{b-1} \leqslant j \leqslant N$, in the $b$ th iteration of the for-loop. Hence, Nicol's algorithm performs $O(\log N) P R O B E$ calls to find $i_{b}$ at iteration $b$, and each PROBE call costs $O(P \log (N / P))$. Thus, the cost of computing an individual $B_{b}$ value is $O(P \log N \log (N / P))$. Since $P-$ 1 such $B_{b}$ values are computed, the overall complexity of Nicol's algorithm is $O\left(N+P^{2} \log N \log (N / P)\right)$, where $O(N)$ cost comes from the initial prefix-sum operation on $\mathcal{W}$.

Two possible implementations of Nicol's algorithm are presented in Fig. 5. Fig. 5(a) illustrates a straightforward implementation, whereas Fig. 5(b) illustrates a careful implementation, which maintains and uses the information from previous probes to answer without calling the PROBE function. As seen in Fig. 5(b), this information is efficiently maintained as an undetermined bottleneck-value range $(L B, U B)$, which is dynamically refined after each probe. Any bottleneck value encountered outside the current range is immediately accepted or rejected. Although this simple scheme does not improve the asymptotic complexity of the algorithm, it drastically reduces the number of probes, as discussed in Section 6.

\section{Proposed CCP algorithms}

In this section, we present proposed methods. First, we describe how to bound the separator indices for an optimal solution to reduce the search space. Then, we show how this technique can be used to improve the performance of the dynamic programming algorithm. We continue with our discussion on improving the MS algorithm, and propose a novel refinement algorithm, which we call the bidding algorithm. Finally, we discuss parametric search methods, proposing improvements for the bisection and Nicol's algorithms.

\subsection{Restricting the search space}

Our proposed CCP algorithms exploit lower and upper bounds on the optimal bottleneck value to restrict 


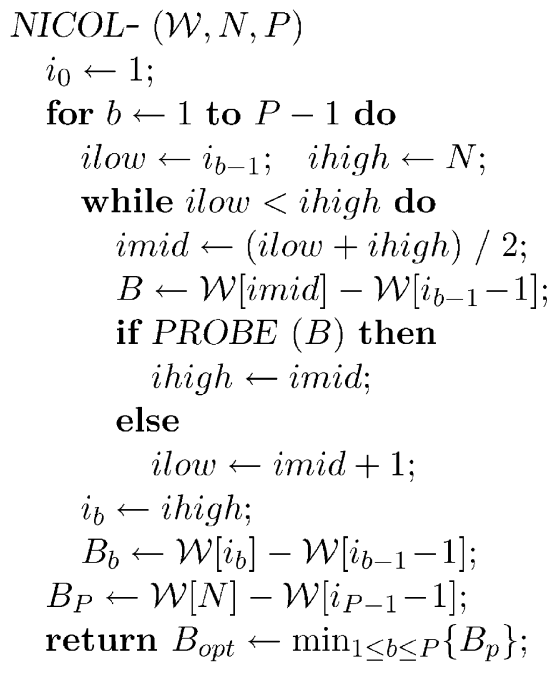

(a)

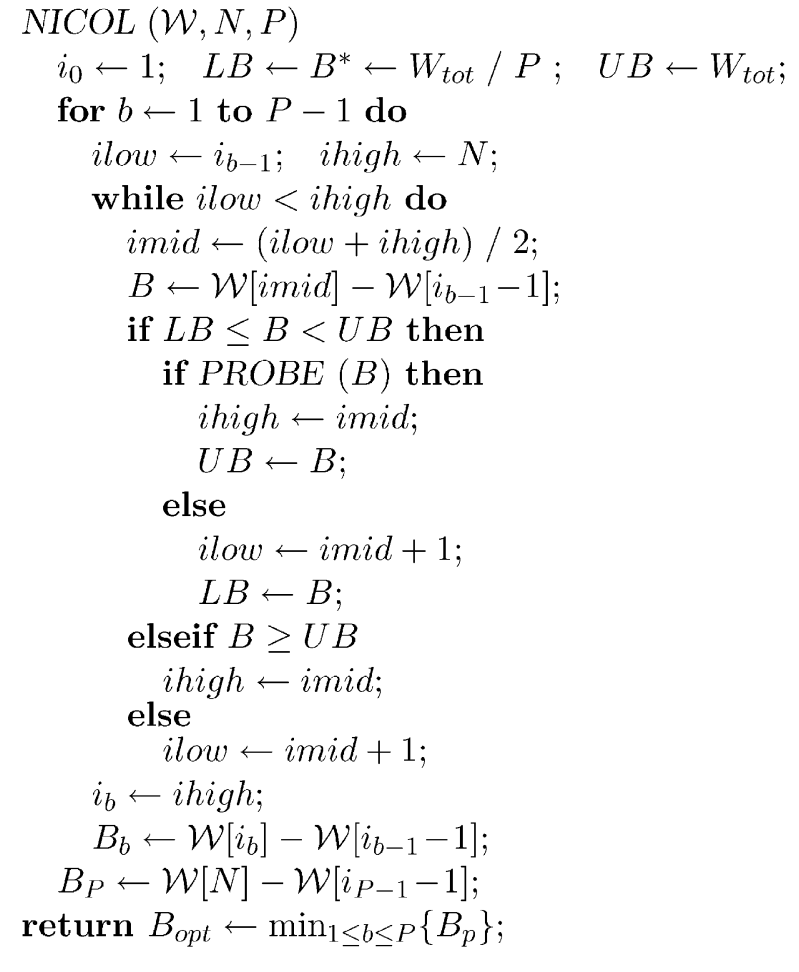

(b)

Fig. 5. Nicol's [27] algorithm: (a) straightforward implementation, (b) careful implementation with dynamic bottleneck-value bounding.

the search space for separator values as a preprocessing step. Natural lower and upper bounds for the optimal bottleneck value $B_{\text {opt }}$ of a given CCP problem instance $(\mathcal{W}, N, P) \quad$ are $\quad L B=\max \left\{B^{*}, w_{\max }\right\} \quad$ and $\quad U B=$ $B^{*}+w_{\max }$, respectively, where $B^{*}=W_{\text {tot }} / P$. Since $w_{\max }<B^{*}$ in coarse grain parallelization of most realworld applications, our presentation will be for $w_{\max }<B^{*}=L B$ even though all results are valid when $B^{*}$ is replaced with $\max \left\{B^{*}, w_{\max }\right\}$. The following lemma describes how to use these natural bounds on $B_{\text {opt }}$ to restrict the search space for the separator values.

Lemma 4.1. For a given CCP problem instance $(\mathcal{W}, N, P)$, if $B_{\mathrm{f}}$ is a feasible bottleneck value in the range $\left[B^{*}, B^{*}+w_{\max }\right]$, then there exists a partition $\Pi=$ $\left\langle s_{0}, s_{1}, \ldots, s_{P}\right\rangle$ of cost $C(\Pi) \leqslant B_{\mathrm{f}}$ with $S L_{p} \leqslant s_{p} \leqslant S H_{p}$, for $1 \leqslant p<P$, where $S L_{p}$ and $S H_{p}$ are, respectively, the smallest and largest indices such that

$$
\begin{aligned}
& W_{1, S L_{p}} \geqslant p\left(B^{*}-w_{\max }(P-p) / P\right) \quad \text { and } \\
& W_{1, S H_{p}} \leqslant p\left(B^{*}+w_{\max }(P-p) / P\right) .
\end{aligned}
$$

Proof. Let $B_{\mathrm{f}}=B^{*}+w$, where $0 \leqslant w<w_{\max }$. Partition $\Pi$ can be constructed by $\operatorname{PROBE}(B)$, which loads the first $p$ processors as much as possible subject to $L_{q} \leqslant B_{\mathrm{f}}$, for $q=1,2, \ldots, p$. In the worst case, $w_{s_{p}+1}=w_{\max }$ for each of the first $p$ processors. Thus, we have $W_{1, s_{p}} \geqslant f(w)=$ $p\left(B^{*}+w-w_{\max }\right)$ for $p=1,2, \ldots, P-1$. However, it should be possible to divide the remaining subchain $\mathcal{T}_{s_{p}+1, N}$ into $P-p$ parts without exceeding $B_{\mathrm{f}}$, i.e., $W_{s_{p+1}, N} \leqslant(P-p)\left(B^{*}+w\right)$. Thus, we also have $W_{1, s_{p}} \geqslant g(w)=W_{\text {tot }}-(P-p)\left(B^{*}+w\right)$. Note that $f(w)$ is an increasing function of $w$, whereas $g(w)$ is a decreasing function of $w$. The minimum of $\max \{f(w), g(w)\}$ is at the intersection of $f(w)$ and $g(w)$ so that $W_{1, s_{p}} \geqslant p\left(B^{*}-w_{\max }(P-p) / P\right)$.

To prove the upper bounds, we can start with $W_{1, s_{p}} \leqslant f(w)=p\left(B^{*}+w\right)$, which holds when $L_{q}=B^{*}+$ $w$ for $q=1, \ldots, p$. The condition $W_{s_{p}+1, N} \geqslant(P-p)\left(B^{*}+\right.$ $\left.w-w_{\max }\right)$, however, ensures feasibility of $B_{\mathrm{f}}=B^{*}+w$, since $P R O B E(B)$ can always load each of the remaining $(P-p)$ processors with $B^{*}+w-w_{\max }$. Thus, we also have $W_{1, s_{p}} \leqslant g(w)=W_{\text {tot }}-(P-p)\left(B^{*}+w-w_{\max }\right)$. Here, $f(w)$ is an increasing function of $w$, whereas $g(w)$ is a decreasing function of $w$, which yields $W_{1, s_{p}} \leqslant p\left(B^{*}+w_{\max }(P-p) / P\right)$.

Corollary 4.2. The separator range weights are $\Delta W_{p}=$ $\sum_{i=S L_{p}}^{S H_{p}} w_{i}=W_{1, S H_{p}}-W_{1, S L_{p}}=2 w_{\max } p(P-p) / P$ with $a$ maximum value $P w_{\max } / 2$ at $p=P / 2$.

Applying this corollary requires finding $w_{\max }$, which entails an overhead equivalent to that of the prefix-sum operation, and hence should be avoided. In this work, we adopt a practical scheme to construct the bounds on separator indices. We run the $R B$ heuristic to find a 
hopefully good bottleneck value $B_{R B}$, and use $B_{R B}$ as an upper bound for bottleneck values, i.e., $U B=B_{R B}$. Then we run $L R-P R O B E\left(B_{R B}\right)$ and $R L-P R O B E\left(B_{R B}\right)$ to construct two mappings $\Pi^{1}=\left\langle h_{0}^{1}, h_{1}^{1}, \ldots, h_{P}^{1}\right\rangle$ and $\Pi^{2}=\left\langle\ell_{0}^{2}, \ell_{1}^{2}, \ldots, \ell_{P}^{2}\right\rangle$ with $C\left(\Pi^{1}\right), C\left(\Pi^{2}\right) \leqslant B_{R B}$. Here, $L R-P R O B E$ denotes the left-to-right probe given in Fig. 3, whereas $R L-P R O B E$ denotes a right-to-left probe function, which can be considered as the dual of the $L R-P R O B E$. $R L-P R O B E$ exploits the greedy-choice property from right to left. That is, $R L-P R O B E$ assigns subchains from the right end towards the left end of the task chain to processors in the order $\mathcal{P}_{P}, \mathcal{P}_{P-1}, \ldots, \mathcal{P}_{1}$. From these two mappings, lower and upper bound values for $s_{p}$ separator indices are constructed as $S L_{p}=$ $\ell_{p}^{2}$ and $S H_{p}=h_{p}^{1}$, respectively. These bounds are further refined by running $L R-P R O B E\left(B^{*}\right)$ and $R L-P R O B E\left(B^{*}\right)$ to construct two mappings $\Pi^{3}=$ $\left\langle\ell_{0}^{3}, \ell_{1}^{3}, \ldots, \ell_{P}^{3}\right\rangle$ and $\Pi^{4}=\left\langle h_{0}^{4}, h_{1}^{4}, \ldots, h_{P}^{4}\right\rangle$, and then defining $S L_{p}=\max \left\{S L_{p}, \ell_{p}^{3}\right\}$ and $S H_{p}=\min \left\{S H_{p}, h_{p}^{4}\right\}$ for $1 \leqslant p<P$. Lemmas 4.3 and 4.4 prove correctness of these bounds.

Lemma 4.3. For a given $C C P$ problem instance $(\mathcal{W}, N, P)$ and a feasible bottleneck value $B_{\mathrm{f}}$, let $\Pi^{1}=$ $\left\langle h_{0}^{1}, h_{1}^{1}, \ldots, h_{P}^{1}\right\rangle$ and $\Pi^{2}=\left\langle\ell_{0}^{2}, \ell_{1}^{2}, \ldots, \ell_{P}^{2}\right\rangle$ be the partitions constructed by $L R-P R O B E\left(B_{\mathrm{f}}\right)$ and $R L-P R O B E\left(B_{\mathrm{f}}\right)$, respectively. Then any partition $\Pi=\left\langle s_{0}, s_{1}, \ldots, s_{P}\right\rangle$ of cost $C(\Pi)=B \leqslant B_{\mathrm{f}}$ satisfies $\ell_{p}^{2} \leqslant s_{p} \leqslant h_{p}^{1}$.

Proof. By the property of $\operatorname{LR}-\operatorname{PROBE}\left(B_{\mathrm{f}}\right), h_{p}^{1}$ is the largest index such that $\mathcal{T}_{1, h_{p}^{1}}$ can be partitioned into $p$ parts without exceeding $B_{\mathrm{f}}$. If $s_{p}>h_{p}^{1}$, then the bottleneck value will exceed $B_{\mathrm{f}}$ and thus $B$. By the property of $R L-P R O B E\left(B_{\mathrm{f}}\right), \ell_{p}^{2}$ is the smallest index where $\mathcal{T}_{\ell_{p}^{2}, N}$ can be partitioned into $P-p$ parts without exceeding $B_{\mathrm{f}}$. If $s_{p}<\ell_{p}^{2}$, then the bottleneck value will exceed $B_{\mathrm{f}}$ and thus $B$.

Lemma 4.4. For a given CCP problem instance $(\mathcal{W}, N, P)$, let $\Pi^{3}=\left\langle\ell_{0}^{3}, \ell_{1}^{3}, \ldots, \ell_{P}^{3}\right\rangle$ and $\Pi^{4}=$ $\left\langle h_{0}^{4}, h_{1}^{4}, \ldots, h_{P}^{4}\right\rangle$ be the partitions constructed by $\operatorname{LR}-P R O B E\left(B^{*}\right)$ and $R L-P R O B E\left(B^{*}\right)$, respectively. Then for any feasible bottleneck value $B_{\mathrm{f}}$, there exists a partition $\Pi=\left\langle s_{0}, s_{1}, \ldots, s_{P}\right\rangle$ of cost $C(\Pi) \leqslant B_{\mathrm{f}}$ that satisfies $\ell_{p}^{3} \leqslant s_{p} \leqslant h_{p}^{4}$.

Proof. Consider the partition $\Pi=\left\langle s_{0}, s_{1}, \ldots, s_{P}\right\rangle$ constructed by $\operatorname{LR}-P R O B E\left(B_{\mathrm{f}}\right)$. It is clear that this partition already satisfies the lower bounds, i.e., $s_{p} \geqslant \ell_{p}^{3}$. Assume $s_{p}>h_{p}^{4}$, then partition $\Pi^{\prime}$ obtained by moving $s_{p}$ back to $h_{p}^{4}$ also yields a partition with cost $C\left(\Pi^{\prime}\right) \leqslant B_{\mathrm{f}}$, since $\mathcal{T}_{h_{p}^{4}+1, N}$ can be partitioned into $P-p$ parts without exceeding $B^{*}$.

The difference between Lemmas 4.3 and 4.4 is that the former ensures the existence of all partitions with $\operatorname{cost} \leqslant B_{\mathrm{f}}$ within the given separator-index ranges, whereas the latter ensures only the existence of at least one such partition within the given ranges. The following corollary combines the results of these two lemmas.

Corollary 4.5. For a given CCP problem instance $(\mathcal{W}, N, P)$ and a feasible bottleneck value $B_{\mathrm{f}}$, let $\Pi^{1}=\left\langle h_{0}^{1}, h_{1}^{1}, \ldots, h_{P}^{1}\right\rangle, \quad \Pi^{2}=\left\langle\ell_{0}^{2}, \ell_{1}^{2}, \ldots, \ell_{P}^{2}\right\rangle, \quad \Pi^{3}=$ $\left\langle\ell_{0}^{3}, \ell_{1}^{3}, \ldots, \ell_{P}^{3}\right\rangle$, and $\Pi^{4}=\left\langle h_{0}^{4}, h_{1}^{4}, \ldots, h_{P}^{4}\right\rangle$ be the partitions constructed by $L R-P R O B E\left(B_{\mathrm{f}}\right), R L-P R O B E\left(B_{\mathrm{f}}\right)$, $L R-P R O B E\left(B^{*}\right)$, and $R L-P R O B E\left(B^{*}\right)$, respectively. Then for any feasible bottleneck value $B$ in the range $\left[B^{*}, B_{\mathrm{f}}\right]$, there exists a partition $\Pi=\left\langle s_{0}, s_{1}, \ldots, s_{P}\right\rangle$ of cost $C(\Pi) \leqslant B$ with $S L_{p} \leqslant s_{p} \leqslant S H_{p}$, for $1 \leqslant p<P$, where $S L_{p}=\max \left\{\ell_{p}^{2}, \ell_{p}^{3}\right\}$ and $S H_{p}=\min \left\{h_{p}^{1}, h_{p}^{4}\right\}$.

Corollary 4.6. The separator range weights become $\Delta W_{p}=2 \min \{p,(P-p)\} w_{\max }$ in the worst case, with a maximum value $P w_{\max }$ at $p=P / 2$.

Lemma 4.1 and Corollary 4.5 infer the following theorem since $B^{*} \leqslant B_{\text {opt }} \leqslant B^{*}+w_{\max }$.

Theorem 4.7. For a given CCP problem instance $(\mathcal{W}, N, P)$, and $S L_{p}$ and $S H_{p}$ index bounds constructed according to Lemma 4.1 or Corollary 4.5, there exists an optimal partition $\Pi_{\mathrm{opt}}=\left\langle s_{0}, s_{1}, \ldots, s_{P}\right\rangle$ with $S L_{p} \leqslant$ $s_{p} \leqslant S H_{p}$, for $1 \leqslant p<P$.

Comparison of separator range weights in Lemma 4.1 and Corollary 4.5 shows that separator range weights produced by the practical scheme described in Corollary 4.5 may be worse than those of Lemma 4.1 by a factor of two. This is only the worst-case behavior however, and the practical scheme normally finds much better bounds, since order in the chain usually prevents the worst-case behavior and $B_{R B}<B^{*}+w_{\max }$. Experimental results in Section 6 justify this expectation.

\subsubsection{Complexity analysis models}

Corollaries 4.2 and 4.6 give bounds on the weights of the separator-index ranges. However, we need bounds on the sizes of these separator-index ranges for computational complexity analysis of the proposed CCP algorithms. Here, the size $\Delta S_{p}=S H_{p}-S L_{p}+1$ denotes the number of tasks within the $p$ th range $\left[S L_{p}, S H_{p}\right]$. Miguet and Pierson [24] propose the model $w_{i}=\theta\left(w_{\text {avg }}\right)$ for $i=1,2, \ldots, N$ to prove that their $H 1$ and $H 2$ heuristics allocate $\theta(N / P)$ tasks to each processor, where $w_{\text {avg }}=W_{\text {tot }} / N$ is the average task weight. This assumption means that the weight of each task is not too far away from the average task weight. Using Corollaries 4.2 and 4.5 , this model induces $\Delta S_{p}=$ $O\left(P w_{\max } / w_{\text {avg }}\right)$. Moreover, this model can be exploited to induce the optimistic bound $\Delta S_{p}=O(P)$. However, 
we find their model too restrictive, since the minimum and maximum task weights can deviate substantially from $w_{\text {avg }}$. Here, we establish a looser and more realistic model on task weights so that for any subchain $\mathcal{T}_{i, j}$ with weight $W_{i, j}$ sufficiently larger than $w_{\max }$, the average task weight within subchain $\mathcal{T}_{i, j}$ satisfies $\Omega\left(w_{\text {avg }}\right)$. That is, $\Delta_{i, j}=j-i+1=O\left(W_{i, j} / w_{\text {avg }}\right)$. This model, referred to here as model $\mathcal{M}$, directly induces $\Delta S_{p}=O\left(P w_{\max } / w_{\text {avg }}\right)$, since $\Delta W_{p} \leqslant \Delta W_{P / 2}=P w_{\max } / 2$ for $p=1,2, \ldots, P-1$.

\subsection{Dynamic-programming algorithm with static separator-index bounding}

The proposed DP algorithm, referred to here as the $\mathrm{DP}+$ algorithm, exploits bounds on the separator indices for an efficient solution. Fig. 6 illustrates the proposed DP + algorithm, where input parameters $S L$ and $S H$ denote the index bound arrays, each of size $P$, computed according to Corollary 4.5 with $B_{\mathrm{f}}=B_{R B}$. Note that $S L_{P}=S H_{P}=N$, since only $B[P, N]$ needs to be computed in the last row. As seen in Fig. 6, only $B_{j}^{p}$ values for $j=S L_{p}, S L_{p}+1, \ldots, S H_{p}$ are computed at each row $p$ by exploiting Corollary 4.5 , which ensures existence of an optimal partition $\Pi_{\mathrm{opt}}=\left\langle s_{0}, s_{1}, \ldots, s_{P}\right\rangle$ with $S L_{p} \leqslant s_{p} \leqslant S H_{p}$. Thus, these $B_{j, j}^{p}$ values will suffice for correct computation of $B_{i}^{p+1}$ values for $i=$ $S L_{p+1}, S L_{p+1}+1, \ldots, S H_{p+1}$ at the next row $p+1$.

As seen in Fig. 6, explicit range checking is avoided in this algorithm for utmost efficiency. However, the $j$ index may proceed beyond $S H_{p}$ to $S H_{p}+1$ within the repeat-until-loop while computing $B_{i}^{p+1}$ with $S L_{p+1} \leqslant i \leqslant S H_{p+1}$ in two cases. In both cases, functions $W_{j+1, i}$ and $B_{j}^{p}$ intersect in the open interval $\left(S H_{p}, S H_{p}+\right.$ 1) so that $B_{S H_{p}}^{p}<W_{S H_{p}+1, i}$ and $B_{S H_{p}+1}^{p} \geqslant W_{S H_{p}+2, i}$. In the first case, $i=S H_{p}+1$ so that $W_{j+1, i}$ and $B_{j}^{p}$ intersect in

$$
\begin{aligned}
& D P+(\mathcal{W}, N, P, S L, S H) \\
& B[1, i] \leftarrow \mathcal{W}[i] \text { for } i=S L_{1}, S L_{1}+1, \ldots, S H_{1} ; \\
& B\left[1, S H_{1}+1\right] \leftarrow \infty ; \\
& \text { for } p \leftarrow 2 \text { to } P \text { do } \\
& \quad j \leftarrow S L_{p-1} ; \\
& \quad \text { for } i \leftarrow S L_{p} \text { to } S H_{p} \text { do } \\
& \quad \text { if } \mathcal{W}[i]-\mathcal{W}[j]>B[p-1, j] \text { then } \\
& \quad \text { repeat } j \leftarrow j+1 \text { until } \mathcal{W}[i]-\mathcal{W}[j] \leq B[p-1, j] ; \\
& \quad \text { if } \mathcal{W}[i]-\mathcal{W}[j-1]<B[p-1, j] \text { then } \\
& \quad j \leftarrow j-1 ; \\
& \quad B[p, i] \leftarrow \mathcal{W}[i]-\mathcal{W}[j] ; \\
& \quad \text { else } \\
& \quad B[p, i] \leftarrow B[p-1, j] ; \\
& \quad \text { else } \\
& \quad B[p, i] \leftarrow B[p-1, j] ; \\
& B\left[1, S H_{p}+1\right] \leftarrow \infty ; \\
& \text { return } B_{\text {opt }} \leftarrow B[P, N] ;
\end{aligned}
$$

Fig. 6. Dynamic-programming algorithm with static separator-index bounding. $(i-1, i)$, which implies that $B_{i}^{p+1}=W_{i-1, i}$, with $j_{i}^{p+1}=$ $S L_{p}$, since $W_{i-1, i}<B_{i}^{p}$, as mentioned in Section 3.2. In the second case, $i>S H_{p}+1$, for which Corollary 4.5 guarantees that $B_{i}^{p+1}=W_{S L_{p}+1, i} \leqslant B_{S H_{p}+1}^{p}$, and thus we can safely select $j_{i}^{p+1}=S L_{p}$. Note that $W_{S L_{p}+1, i}=$ $B_{S H_{p}+1}^{p}$ may correspond to a case leading to another optimal partition with $j_{i}^{p+1}=s_{p+1}=S H_{p}+1$. As seen in Fig. 6, both cases are efficiently resolved simply by storing $\infty$ to $B_{S H_{p}+1}^{p}$ as a sentinel. Hence, in such cases, the condition $W_{S H_{p}+1, i}<B_{S H_{p}+1}^{p}=\infty$ in the if-then statement following the repeat-until-loop is always true so that the $j$-index automatically moves back to $S H_{p}$. The scheme of computing $B_{S H_{p}+1}^{p}$ for each row $p$, which seems to be a natural solution, does not work since correct computation of $B_{S H_{p+1}+1}^{p+1}$ may necessitate more than one $B_{j}^{p}$ value beyond the $S H_{p}$ index bound.

A nice feature of the DP approach is that it can be used to generate all optimal partitions by maintaining a $P \times N$ matrix to store the minimum $j_{i}^{p}$ index values defining the $B_{i}^{p}$ values at the expense of increased execution time and increased asymptotic space requirement. Recall that index bounds $S L$ and $S H$ computed according to Corollary 4.5 restrict the search space for at least one optimal solution. The index bounds can be computed according to Lemma 4.4 for this purpose, since the search space restricted by Lemma 4.4 includes all optimal solutions.

The running time of the proposed $\mathrm{DP}+$ algorithm is $O(N+P \log N)+\sum_{p=1}^{P} \theta\left(\Delta S_{p}\right)$. Here, $O(N)$ cost comes from the initial prefix-sum operation on the $\mathcal{W}$ array, and $O(P \log N)$ cost comes from the running time of the $R B$ heuristic and computing the separator-index bounds $S L$ and $S H$ according to Corollary 4.5. Under model $\mathcal{M}, \Delta S_{p}=O\left(P w_{\max } / w_{\text {avg }}\right)$, and hence the complexity is $O\left(N+P \log N+P^{2} w_{\max } / w_{\text {avg }}\right)$. The algorithm becomes linear in $N$ when the separator-index ranges do not overlap, which is guaranteed by the condition $w_{\max }=O\left(2 W_{\mathrm{tot}} / P^{2}\right)$.

\subsection{Iterative refinement algorithms}

In this work, we improve the MS algorithm and propose a novel $\mathrm{CCP}$ algorithm, namely the bidding algorithm, which is run-time efficient for small-tomedium number of processors. The main difference between the MS and the bidding algorithms is as follows: the MS algorithm moves along a series of feasible bottleneck values, whereas the bidding algorithm moves along a sequence of infeasible bottleneck values so that the first feasible bottleneck value becomes the optimal value.

\subsubsection{Improving the MS algorithm}

The performance of the MS algorithm strongly depends on the initial partition. The initial partition proposed by Manne and Sørevik [23] satisfies the leftist 
partition constraint, but it leads to very poor run-time performance. Here, we propose using the partition generated by $\operatorname{PROBE}\left(B^{*}\right)$ as an initial partition. This partition is also a leftist partition, since moving any separator to the left will not decrease the load of the bottleneck processor. This simple observation leads to significant improvement in run-time performance of the algorithm. Also, using a heap as a priority queue does not give better run-time performance than using a running maximum despite its superior asymptotic complexity. In our implementation, we use a running maximum.

\subsubsection{Bidding algorithm}

This algorithm increases the bottleneck value gradually, starting from the ideal bottleneck value $B^{*}$, until it finds a feasible partition, which is also optimal. Consider a partition $\Pi_{t}=\left\langle s_{0}, s_{1}, \ldots, s_{P}\right\rangle$ constructed by $\operatorname{PROBE}\left(B_{t}\right)$ for an infeasible $B_{t}$. After detecting the infeasibility of this $B_{t}$ value, the point is to determine the next larger bottleneck value $B$ to be investigated. Clearly, the separator indices of the partitions to be constructed by future $P R O B E(B)$ calls with $B>B_{t}$ will never be to the left of the respective separator indices of $\Pi_{t}$. Moreover, at least one of the separators should move right for feasibility, since the load of the last processor determines infeasibility of the current $B_{t}$ value (i.e., $L_{P}>B_{t}$ ). To avoid missing the smallest feasible bottleneck value, the next larger $B$ value is selected as the minimum of processor loads that will be obtained by moving the end-index of every processor to the right by one position. That is, the next larger $B$ value is equal to $\min \left\{\min _{1 \leqslant p<P}\left\{L_{p}+w_{s_{p}+1}\right\}, L_{P}\right\}$. Here, we call the $L_{p}+w_{s_{p}+1}$ value the bid of processor $\mathcal{P}_{p}$, which refers to the load of $\mathcal{P}_{p}$ if the first task $t_{s_{p}+1}$ of the next processor is augmented to $\mathcal{P}_{p}$. The bid of the last processor $\mathcal{P}_{P}$ is equal to the load of the remaining tasks. If the smallest bid $B$ comes from processor $\mathcal{P}_{b}$, probing with new $B$ is performed only for the remaining processors $\left\langle\mathcal{P}_{b}, \mathcal{P}_{b+1}, \ldots, \mathcal{P}_{P}\right\rangle$ in the suffix $\mathcal{W}_{s_{b-1}+1: N}$ of the $\mathcal{W}$ array.

The bidding algorithm is presented in Fig. 7. The innermost while-loop implements a linear probing scheme, such that the new positions of the separators are determined by moving them to the right, one by one. This linear probing scheme is selected because new positions of separators are likely to be in a close neighborhood of previous ones. Note that binary search is used only for setting the separator indices for the first time. After the separator index $s_{p}$ is set for processor $\mathcal{P}_{p}$ during linear probing, the repeat-until-loop terminates if it is not possible to partition the remaining subchain $\mathcal{T}_{s_{p}+1, N}$ into $P-p$ processors without exceeding the current $B$ value, i.e., $r b i d=L_{r} /(P-p)>B$, where $L_{r}$ denotes the weight of the remaining subchain. In this case, the next larger $B$ value is determined by considering the best bid among the first $p$ processors and rbid.

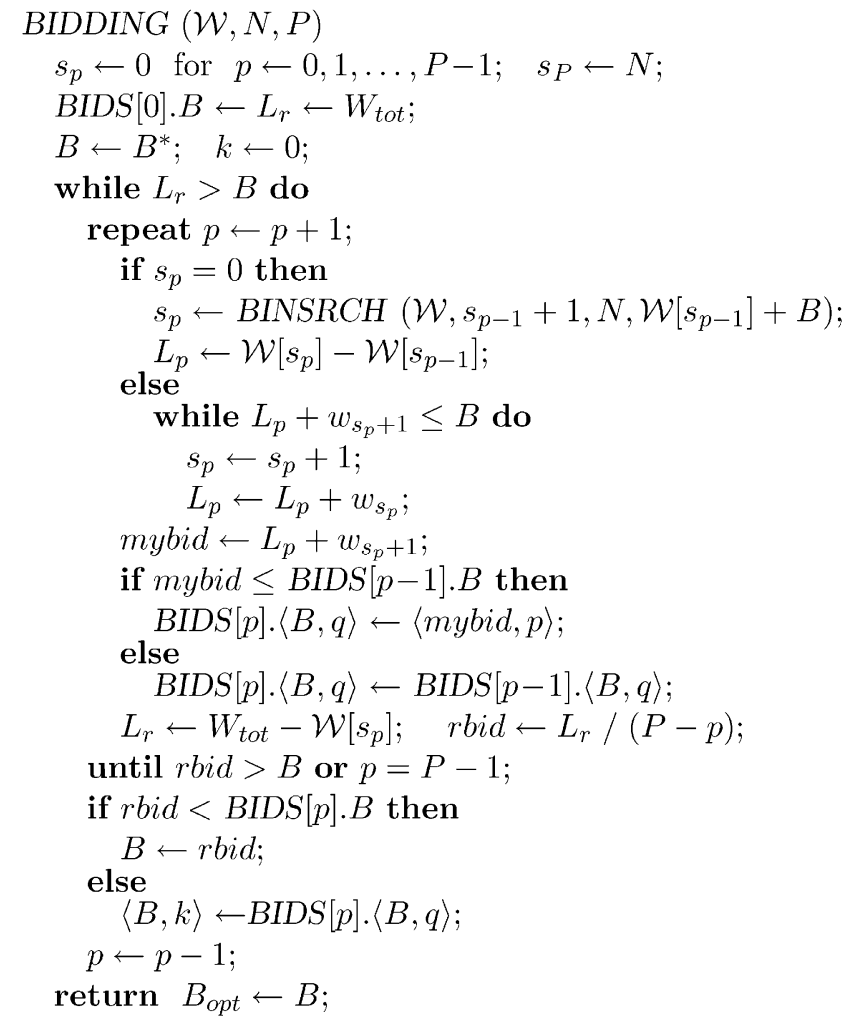

Fig. 7. Bidding algorithm.

As seen in Fig. 7, we maintain a prefix-minimum array $B I D S$ for computing the next larger $B$ value. Here, $B I D S$ is an array of records of length $P$, where $B I D S[p] . B$ and $B I D S[p] . b$ store the best bid value of the first $p$ processors and the index of the defining processor, respectively. BIDS[0] helps the correctness of the running prefix-minimum operation.

The complexity of the bidding algorithm for integer task weights under model $\mathcal{M}$ is $O(N+P \log N+$ $\left.P w_{\max }+P^{2}\left(w_{\max } / w_{\text {avg }}\right)\right)$. Here, $O(N)$ cost comes from the initial prefix-sum operation on the $\mathcal{W}$ array, and $O(P \log N)$ cost comes from initial settings of separators through binary search. The $B$ value is increased at most $B_{\text {opt }}-B^{*}<w_{\max }$ times, and each time the next $B$ value can be computed in $O(P)$ time, which induces the cost $O\left(P w_{\max }\right)$. The total area scanned by the separators is at most $O\left(P^{2}\left(w_{\max } / w_{\text {avg }}\right)\right)$. For noninteger task weights, complexity can reach $O\left(P \log N+P^{3}\left(w_{\max } / w_{\text {avg }}\right)\right)$ in the worst case, which occurs when only one separator index moves to the right by one position at each $B$ value. We should note here that using a min-heap for finding the next $B$ value enables terminating a repeat-loop iteration as soon as a separator-index does not move. The trade-off in this scheme is the $O(\log P)$ cost incurred at each separator-index move due to respective key-update operations on the heap. We implemented this scheme as well, but observed increased execution times. 


\subsection{Parametric search algorithms}

In this work, we apply theoretical findings given in Section 4.1 for an improved probe algorithm. The improved algorithm, which we call the restricted probe $(R P R O B E)$, exploits bounds computed according to Corollary 4.5 (with $B_{\mathrm{f}}=B_{R B}$ ) to restrict the search space for $s_{p}$ separator values during binary searches on the $\mathcal{W}$ array. That is, $\operatorname{BINSRCH}\left(\mathcal{W}, S L_{p}, S H_{p}, B\right.$ sum $)$ in $R P R O B E$ searches $\mathcal{W}$ in the index range $\left[S L_{p}, S H_{p}\right]$ to find the index $S L_{p} \leqslant s_{p} \leqslant S H_{p}$ such that $\mathcal{W}\left[s_{p}\right] \leqslant B s u m$ and $\mathcal{W}\left[s_{p}+1\right]>$ Bsum via binary search. This scheme and Corollaries 4.2 and 4.6 reduce the complexity of an individual probe to $\sum_{p=1}^{P} \theta\left(\log \Delta_{p}\right)=O(P \log P+$ $\left.P \log \left(w_{\max } / w_{\text {avg }}\right)\right)$. Note that this complexity reduces to $O(P \log P)$ for sufficiently large $P$ where $P=\Omega\left(w_{\max } /\right.$ $\left.w_{\text {avg }}\right)$. Figs. 8-10 illustrate $R P R O B E$ algorithms tailored for the respective parametric-search algorithms.

\subsubsection{Approximate bisection algorithm with dynamic separator-index bounding}

The proposed bisection algorithm, illustrated in Fig. 8, searches the space of bottleneck values in range $\left[B^{*}, B_{R B}\right]$ as opposed to $\left[B^{*}, W_{\text {tot }}\right]$. In this algorithm, if $\operatorname{PROBE}\left(B_{t}\right)=$ TRUE, then the search space is restricted to $B \leqslant B_{t}$ values, and if $\operatorname{PROBE}\left(B_{t}\right)=$ FALSE, then the search space is restricted to $B>B_{t}$ values. In this work, we exploit this simple observation to propose and develop a dynamic probing scheme that increases the efficiency of successive $P R O B E$ calls by modifying separator index-bounds depending on success and failure of the probes. Let $\Pi_{t}=\left\langle t_{0}, t_{1}, \ldots, t_{P}\right\rangle$ be the partition constructed by $\operatorname{PROBE}\left(B_{t}\right)$. Any future $P R O B E(B)$ call with $B \leqslant B_{t}$ will set the $s_{p}$ indices with $s_{p} \leqslant t_{p}$. Thus, the search space for $s_{p}$ can be restricted to those indices $\leqslant t_{p}$. Similarly, any future $\operatorname{PROBE}(B)$ call with $B \geqslant B_{t}$ will set $s_{p}$ indices $\geqslant t_{p}$. Thus, the search space for $s_{p}$ can be restricted to those indices $\geqslant t_{p}$.

As illustrated in Fig. 8, dynamic update of separatorindex bounds can be performed in $\theta(P)$ time by a forloop over $S L$ or $S H$ arrays, depending on failure or success, respectively, of $R P R O B E\left(B_{t}\right)$. In our implementation, however, this update is efficiently achieved in $O(1)$ time through the pointer assignment $S L \leftarrow \Pi$ or $S H \leftarrow \Pi$ depending on failure or success of RPROBE $\left(B_{t}\right)$.
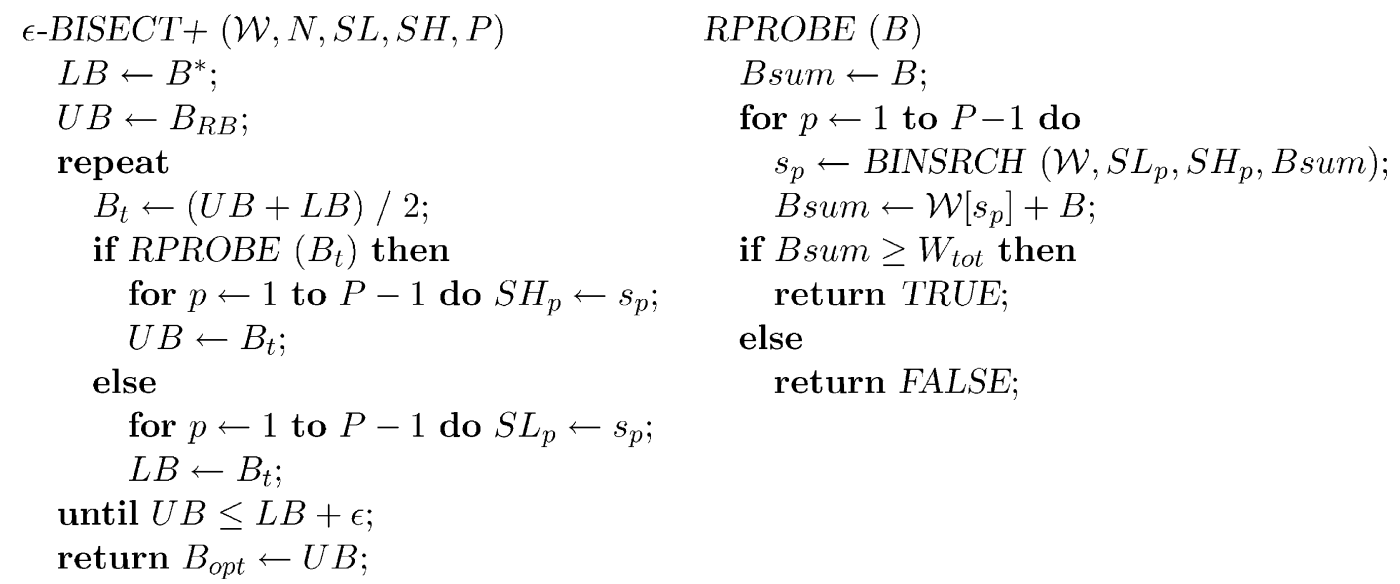

Fig. 8. Bisection as an $\varepsilon$-approximation algorithm with dynamic separator-index bounding.

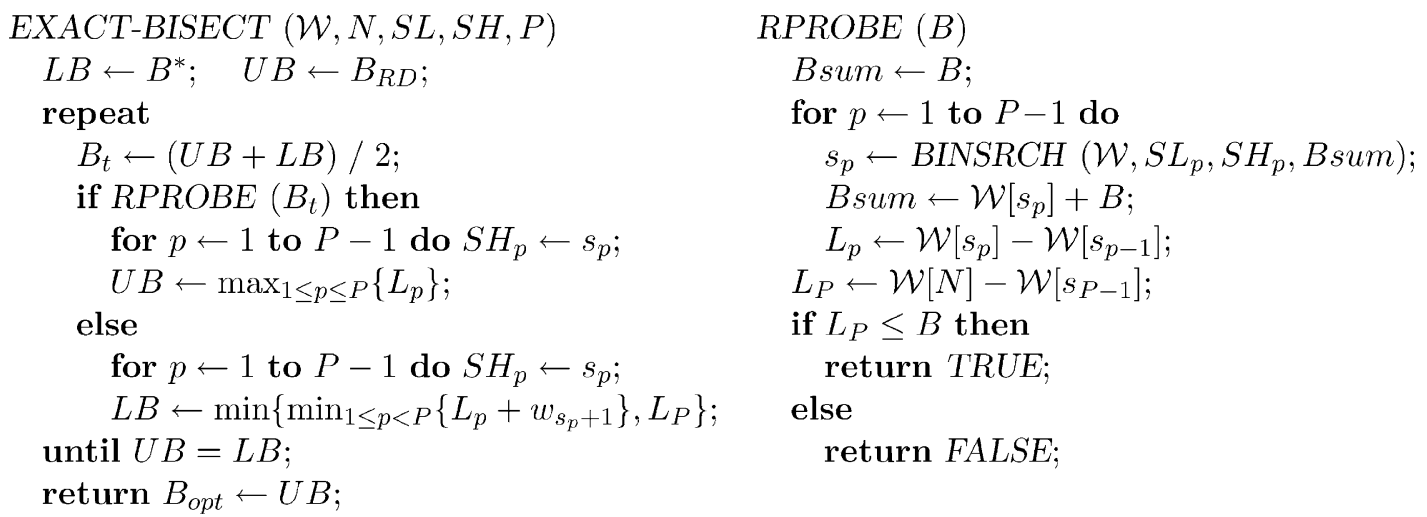

Fig. 9. Exact bisection algorithm with dynamic separator-index bounding. 


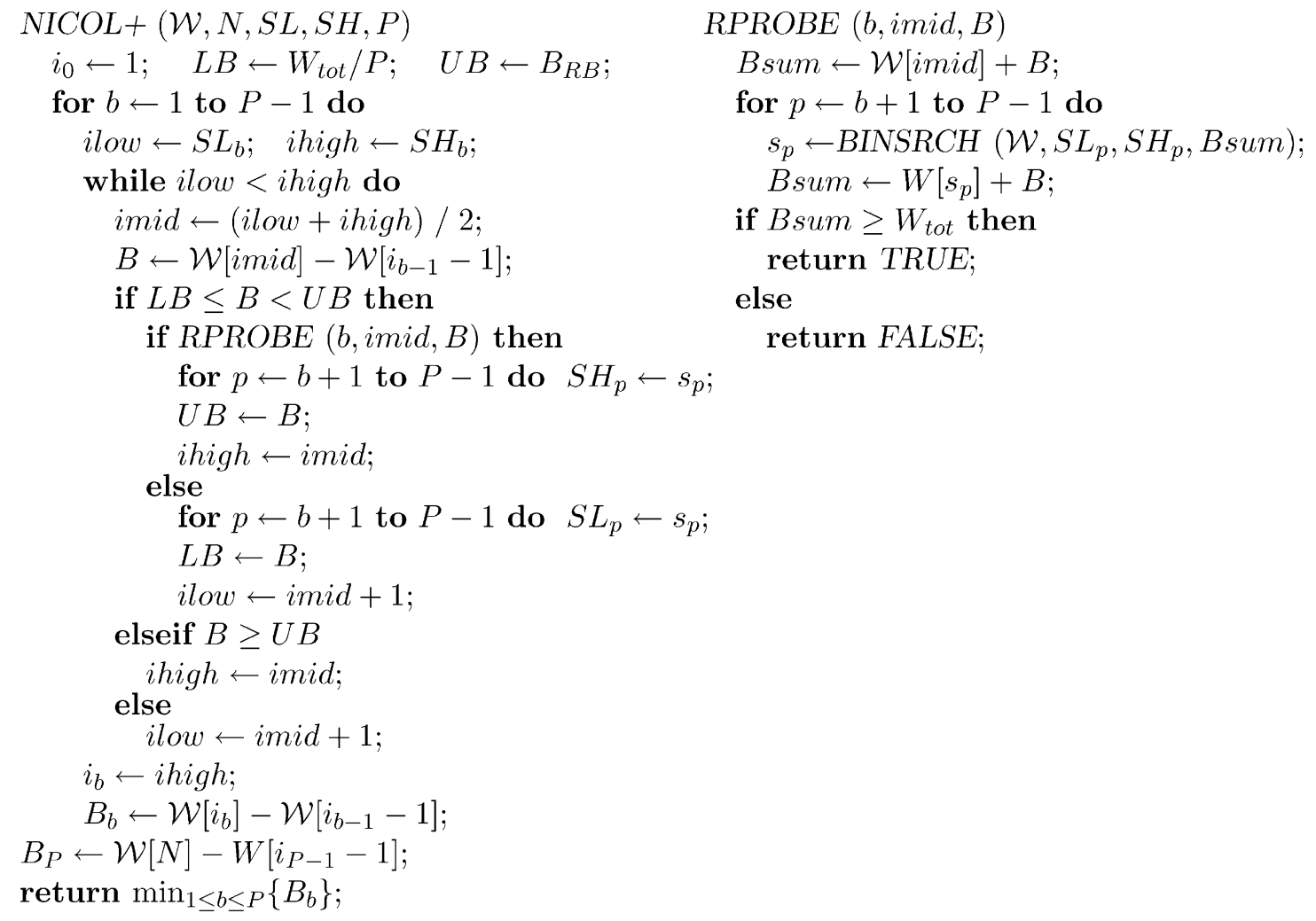

Fig. 10. Nicol's algorithm with dynamic separator-index bounding.

Similar to the $\varepsilon$-BISECT algorithm, the proposed $\varepsilon$-BISECT + algorithm is also an $\varepsilon$-approximation algorithm for general workload arrays. However, both the $\varepsilon$-BISECT and $\varepsilon$-BISECT + algorithms become exact algorithms for integer-valued workload arrays by setting $\varepsilon=1$. As shown in Lemma 3.1, $B_{R B}<B^{*}+w_{\max }$. Hence, for integer-valued workload arrays the maximum number of probe calls in the $\varepsilon$-BISECT + algorithm is $\log w_{\max }$, and thus the overall complexity is $\quad O\left(N+P \log N+\log \left(w_{\max }\right)\left(P \log P+P \log \left(w_{\max } /\right.\right.\right.$ $\left.\left.w_{\text {avg }}\right)\right)$ ) under model $\mathcal{M}$. Here, $O(N)$ cost comes from the initial prefix-sum operation on $\mathcal{W}$ and $O(P \log N)$ cost comes from the running time of the $R B$ heuristic and computing the separator-index bounds $S L$ and $S H$ according to Corollary 4.5.

\subsubsection{Bisection as an exact algorithm}

In this section, we will enhance the bisection algorithm to be an exact algorithm for general workload arrays by clever updating of lower and upper bounds after each probe. The idea is, after each probe moving upper and lower bounds on the value of an optimal solution to a realizable bottleneck value (total weight of a subchain of $\mathcal{W}$ ). This reduces the search space to a finite set of realizable bottleneck values, as opposed to an infinite space of bottleneck values defined by a range $[L B, U B]$. Each bisection step is designed to eliminate at least one candidate value, and thus the algorithm terminates in finite number of steps to find the optimal bottleneck value.

After a probe $R P R O B E\left(B_{t}\right)$, the current upper bound value $U B$ is modified if $R P R O B E\left(B_{t}\right)$ succeeds. Note that RPROBE $\left(B_{t}\right)$ not only determines the feasibility of $B_{t}$, but also constructs a partition $\Pi$ with $\operatorname{cost}\left(\Pi_{t}\right) \leqslant B_{t}$. Instead of reducing the upper bound $U B$ to $B_{t}$, we can further reduce $U B$ to the bottleneck value $B=\operatorname{cost}\left(\Pi_{t}\right) \leqslant B_{t}$ of the partition $\Pi_{t}$ constructed by RPROBE $\left(B_{t}\right)$. Similarly, the current lower bound $L B$ is modified when $R P R O B E\left(B_{t}\right)$ fails. In this case, instead of increasing the bound $L B$ to $B_{t}$, we can exploit the partition $\Pi_{t}$ constructed by $R P R O B E\left(B_{t}\right)$ to increase $L B$ further to the smallest realizable bottleneck value $B$ greater than $B_{t}$. Our bidding algorithm already describes how to compute

$B=\min \left\{\min _{1 \leqslant p<P}\left\{L_{p}+w_{s_{p}+1}\right\}, L_{P}\right\}$,

where $L_{p}$ denotes the load of processor $\mathcal{P}_{p}$ in $\Pi_{t}$. Fig. 9 presents the pseudocode of our algorithm.

Each bisection step divides the set of candidate realizable bottleneck values into two sets, and eliminates one of them. The initial set can have a size between 1 and $N^{2}$. Assuming the size of the eliminated set can be anything between 1 and $N^{2}$, the expected complexity of 
the algorithm is

$$
\begin{aligned}
T(N)= & \frac{1}{N^{2}} \sum_{i=1}^{N^{2}} T(i)+O(P \log P \\
& \left.+P \log \left(w_{\max } / w_{\text {avg }}\right)\right),
\end{aligned}
$$

which has the solution $O(P \log P \log N+$ $\left.P \log N \log \left(w_{\max } / w_{\text {avg }}\right)\right)$. Here, $O\left(P \log P+P \log \left(w_{\max } /\right.\right.$ $\left.\left.w_{\text {avg }}\right)\right)$ is the cost of a probe operation, and $\log N$ is the expected number of probes. Thus, the overall complexity becomes $O\left(N+P \log P \log N+P \log N \log \left(w_{\max } /\right.\right.$ $\left.w_{\text {avg }}\right)$ ), where the $O(N)$ cost comes from the initial prefix-sum operation on the $\mathcal{W}$ array.

\subsubsection{Improving Nicol's algorithm as a divide-and- conquer algorithm}

Theoretical findings of previous sections can be exploited at different levels to improve performance of Nicol's algorithm. A trivial improvement is to use the proposed restricted probe function instead of the conventional one. The careful implementation scheme given in Fig. 5(b) enables use of dynamic separatorindex bounding. Here, we exploit the bisection idea to design an efficient divide-and-conquer approach based on Nicol's algorithm.

Consider the sequence of probes of the form $\operatorname{PROBE}\left(W_{1, j}\right)$ performed by Nicol's algorithm for processor $\mathcal{P}_{1}$ to find the smallest index $j=i_{1}$ such that $\operatorname{PROBE}\left(W_{1, j}\right)=$ TRUE. Starting from a naive bottleneck-value range $\left(L B_{0}=0, U B_{0}=W_{\text {tot }}\right)$, success and failure of these probes can narrow this range to $\left(L B_{1}, U B_{1}\right)$. That is, each $\operatorname{PROBE}\left(W_{1, j}\right)=$ TRUE decreases the upper bound to $W_{1, j}$ and each $\operatorname{PROBE}\left(W_{1, j}\right)=$ FALSE increases the lower bound to $W_{1, j}$. Clearly, we will have $\left(L B_{1}=W_{1, i_{1}-1}, U B_{1}=W_{1, i_{1}}\right)$ at the end of this search for processor $\mathcal{P}_{1}$. Now consider the sequence of probes of the form $\operatorname{PROBE}\left(W_{i_{1}, j}\right)$ performed for processor $\mathcal{P}_{2}$ to find the smallest index $j=i_{2}$ such that $\operatorname{PROBE}\left(W_{i_{1}, j}\right)=$ TRUE. Our key observation is that the partition $\Pi_{t}=$ $\left\langle 0, t_{1}, t_{2}, \ldots, t_{P-1}, N\right\rangle$ to be constructed by any $\operatorname{PROBE}\left(B_{t}\right)$ with $L B_{1}<B_{t}=W_{i_{1}, j}<U B_{1}$ will satisfy $t_{1}=i_{1}-1$, since $W_{1, i_{1}-1}<B_{t}<W_{1, i_{1}}$. Hence, probes with $L B_{1}<B_{t}<U B_{1}$ for processor $\mathcal{P}_{2}$ can be restricted to be performed in $\mathcal{W}_{i_{1}: N}$, where $\mathcal{W}_{i_{1}: N}$ denotes the $\left(N-i_{1}+1\right)$ th suffix of the prefix-summed $\mathcal{W}$ array. This simple yet effective scheme leads to an efficient divide-and-conquer algorithm as follows. Let $\mathcal{T}_{i}^{P-p}$ denote the CCP subproblem of $(P-p)$-way partitioning of the $(N-i+1)$ th suffix $\mathcal{T}_{i, N}=\left\langle t_{i}, t_{i+1}, \ldots, t_{N}\right\rangle$ of the task chain $\mathcal{T}$ onto the $(P-p)$ th suffix $\mathcal{P}_{p, P}=$ $\left\langle\mathcal{P}_{p+1}, \mathcal{P}_{p+2}, \ldots, \mathcal{P}_{P}\right\rangle$ of the processor chain $\mathcal{P}$. Once the index $i_{1}$ for processor $\mathcal{P}_{1}$ is computed, the optimal bottleneck value $B_{\text {opt }}$ can be defined by either $W_{1, i_{1}}$ or the bottleneck value of an optimal $(P-1)$-way partitioning of the suffix subchain $\mathcal{T}_{i_{1}, N}$. That is, $B_{\text {opt }}=$ $\min \left\{B_{1}=W_{1, i_{1}}, C\left(\Pi_{i_{1}}^{P-1}\right)\right\}$. Proceeding in this way, once the indices $\left\langle i_{1}, i_{2}, \ldots, i_{p}\right\rangle$ for the first $p$ processors $\left\langle\mathcal{P}_{1}, \mathcal{P}_{2}, \ldots, \mathcal{P}_{p}\right\rangle$ are determined, then $B_{\text {opt }}=$ $\min \left\{\min _{1 \leqslant b \leqslant p}\left\{B_{b}=W_{i_{b-1}, i_{b}}\right\}, C\left(\Pi_{i_{p}}^{P-p}\right)\right\}$.

This approach is presented in Fig. 10. At the $b$ th iteration of the outer for-loop, given $i_{b-1}, i_{b}$ is found in the inner while-loop by conducting probes on $\mathcal{W}_{i_{b-1}: N}$ to compute $B_{b}=W_{i_{b-1}, i_{b}}$. The dynamic bounds on the separator indices are exploited in two distinct ways according to Theorem 4.7. First, the restricted probe function RPROBE is used for probing. Second, the search spaces for the bottleneck values of the processors are restricted. That is, given $i_{b-1}$, the binary search for $i_{b}$ over all subchain weights of the form $W_{i_{b-1}+1, j}$ for $i_{b-1}<j \leqslant N$ is restricted to $W_{i_{b-1}+1, j}$ values for $S L_{b} \leqslant j \leqslant S H_{b}$.

Under model $\mathcal{M}$, the complexity of this algorithm is $O\left(N+P \log N+w_{\max }\left(P \log P+P \log \left(w_{\max } / w_{\text {avg }}\right)\right)\right)$ for integer task weights, because the number of probes cannot exceed $w_{\max }$, since there are at most $w_{\max }$ distinct bound values in the range $\left[B^{*}, B_{R B}\right]$. For noninteger task weights, the complexity can be given as $O\left(N+P \log N+w_{\max }(P \log P)^{2}+w_{\max } P^{2} \log P \log \left(w_{\max } /\right.\right.$ $\left.\left.w_{\text {avg }}\right)\right)$, since the algorithm makes $O\left(w_{\max } P \log P\right)$ probes. Here, $O(N)$ cost comes from the initial prefix-sum operation on $\mathcal{W}$, and $O(P \log N)$ cost comes from the running time of the $R B$ heuristic and computing the separator-index bounds $S L$ and $S H$ according to Corollary 4.5 .

\section{Load balancing applications}

\subsection{Parallel sparse matrix-vector multiplication}

Sparse matrix-vector multiplication $(\mathrm{SpMxV})$ is one of the most important kernels in scientific computing. Parallelization of repeated $\mathrm{SpMxV}$ computations requires partitioning and distribution of the matrix. Two possible 1D sparse-matrix partitioning schemes are rowwise striping (RS) and columnwise striping (CS). Consider parallelization of $\mathrm{SpMxV}$ operations of the form $y=A x$ in an iterative solver, where $A$ is an $N \times N$ sparse matrix and $y$ and $x$ are $N$-vectors. In $\mathrm{RS}$, processor $\mathcal{P}_{p}$ owns the $p$ th row stripe $A_{p}^{r}$ of $A$ and is responsible for computing $y_{p}=A_{p}^{r} x$, where $y_{p}$ is the $p$ th stripe of vector $y$. In $\mathrm{CS}$, processor $\mathcal{P}_{p}$ owns the $p$ th column stripe $A_{p}^{c}$ of $A$ and is responsible for computing $y^{p}=A_{p}^{c} x$, where $y=\sum_{p=1}^{P} y^{p}$. All vectors used in the solver are divided conformably with row (column) partitioning in the RS (CS) scheme, to avoid unnecessary communication during linear vector operations. RS and $\mathrm{CS}$ schemes require communication before or after local SpMxV computations, thus they can also be considered as pre- and post-communication schemes, respectively. In $\mathrm{RS}$, each task $t_{i} \in \mathcal{T}$ corresponds to the 
atomic task of computing the inner-product of row $i$ of matrix $A$ with column vector $x$. In CS, each task $t_{i} \in \mathcal{T}$ corresponds to the atomic task of computing the sparse $D A X P Y$ operation $y=y+x_{i} a_{* i}$, where $a_{* i}$ is the $i$ th column of $A$. Each nonzero entry in a row and column of A incurs a multiply-and-add operation, thus the computational load $w_{i}$ of task $t_{i}$ is the number of nonzero entries in row $i$ (column $i$ ) in the RS (CS) scheme. This defines how load balancing problem for rowwise and columnwise partitioning of a sparse matrix with a given ordering can be modeled as a CCP problem.

In RS (CS), by allowing only row (column) reordering, load balancing problem can be described as the number partitioning problem, which is NP-Hard [9]. By allowing both row and column reordering, the problem of minimizing communication overhead while maintaining load balance can be described as graph and hypergraph partitioning problems [5,14], which are NP-Hard [10,21] as well. However, possibly high preprocessing overhead involved in these models may not be justified in some applications. If the partitioner is to be used as part of a run-time library for a parallelizing compiler for a data-parallel programming language [33,34], row and column reordering lead to high memory requirement due to the irregular mapping table and extra level of indirection in locating distributed data during each multiply-and-add operation [35]. Furthermore, in some applications, the natural row and column ordering of the sparse matrix may already be likely to induce small communication overhead (e.g., banded matrices).

The proposed CCP algorithms are surprisingly fast so that the initial prefix-sum operation dominates their execution times in sparse-matrix partitioning. In this work, we exploit the standard compressed row storage (CRS) and compressed column storage (CCS) data structures for sparse matrices to avoid the prefix-sum operation. In CRS, an array $D A T A$ of length $N Z$ stores nonzeros of matrix $A$, in row-major order, where $N Z=$ $W_{\text {tot }}$ denotes the total number of nonzeros in $A$. An index array $C O L$ of length $N Z$ stores the column indices of respective nonzeros in array $D A T A$. Another index array $R O W$ of length $N+1$ stores the starting indices of respective rows in the other two arrays. Hence, any subchain weight $W_{i, j}$ can be efficiently computed using $W_{i, j}=R O W[j+1]-R O W[i]$ in $O(1)$ time without any preprocessing overhead. CCS is similar to CRS with rows and columns interchanged, thus $W_{i, j}$ is computed using $W_{i, j}=\operatorname{COL}[j+1]-\operatorname{COL}[i]$.

\subsubsection{A better load balancing model for iterative solvers}

The load balancing problem for parallel iterative solvers has usually been stated considering only the SpMxV computations. However, linear vector operations (i.e., DAXPY and inner-product computations) involved in iterative solvers may have a considerable effect on parallel performance with increasing sparsity of the coefficient matrix. Here, we consider incorporating vector operations into the load-balancing model as much as possible.

For the sake of discussion, we will investigate this problem for a coarse-grain formulation [2,3,32] of the conjugate-gradient (CG) algorithm. Each iteration of CG involves, in order of computational dependency, one $\mathrm{SpMxV}$, two inner-products, and three DAXPY computations. DAXPY computations do not involve communication, whereas inner-product computations necessitate a post global reduction operation [19] on results of the two local inner-products. In rowwise striping, pre-communication operations needed for SpMxV and the global reduction operation constitute pre- and post-synchronization points, respectively, for the aggregate of one local $\mathrm{SpMxV}$ and two local innerproduct computations. In columnwise striping, the global reduction operation in the current iteration and post-communication operations needed for $\mathrm{SpMxV}$ in the next iteration constitute the pre- and post-synchronization points, respectively, for an aggregate of three local DAXPY and one local SpMxV computations. Thus, columnwise striping may be favored for a wider coverage of load balancing. Each vector entry incurs a multiply-and-add operation during each linear vector operation. Hence, DAXPY computations can easily be incorporated into the load-balancing model for the CS scheme by adding a cost of three to the computational weight $w_{i}$ of atomic task $t_{i}$ representing column $i$ of matrix $A$. The initial prefix-sum operation can still be avoided by computing a subchain weight $W_{i, j}$ as $W_{i, j}=$ $C O L[j+1]-C O L[i]+3(j-i+1)$ in constant time. Note that two local inner-product computations still remain uncovered in this balancing model.

\subsection{Sort-first parallel direct volume rendering}

Direct volume rendering (DVR) methods are widely used in rendering unstructured volumetric grids for visualization and interpretation of computer simulations performed for investigating physical phenomena in various fields of science and engineering. A DVR application contains two interacting domains: object space and image space. Object space is a $3 \mathrm{D}$ domain containing the volume data to be visualized. Image space (screen) is a 2D domain containing pixels from which rays are shot into the $3 \mathrm{D}$ object domain to determine the color values of the respective pixels. Based on these domains, there are basically two approaches for data parallel DVR: image- and object-space parallelism, which are also called as sort-first and sort-last parallelism according to the taxonomy based on the point of data redistribution in the rendering pipeline [25]. Pixels or pixel blocks constitute the atomic tasks in sort-first 
parallelism, whereas volume elements (primitives) constitute the atomic tasks in sort-last parallelism.

In sort-first parallel DVR, screen space is decomposed into regions and each region is assigned to a separate processor for local rendering. The primitives, whose projection areas intersect more than one region, are replicated. Sort-first parallelism has an advantage of processors generating complete images for their local screen subregion, but it faces load-balancing problems in the DVR of unstructured grids due to uneven onscreen primitive distribution.

Image-space decomposition schemes for sort-first parallel DVR can be classified as static and adaptive [20]. Static decomposition is a view-independent scheme, and the load-balancing problem is solved implicitly by scattered assignment of pixels or pixel blocks. Loadbalancing performance of this scheme depends on the assumption that neighbor pixels are likely to have equal workload since they are likely to have similar views of the volume. As the scattered assignment scheme assigns adjacent pixels or pixel blocks to different processors, it disturbs image-space coherency and increases the amount of primitive replication. Adaptive decomposition is a view-dependent scheme, and the load-balancing problem is solved explicitly by using the primitive distribution on the screen.

In adaptive image-space decomposition, the number of primitives with bounding-box approximation is taken to be the workload of a screen region. Primitives constituting the volume are tallied to a $2 \mathrm{D}$ coarse mesh superimposed on the screen. Some primitives may intersect multiple cells. The inverse-area heuristic [26] is used to decrease the amount of error due to counting such primitives multiple times. Each primitive increments the weight of each cell it intersects by a value inversely proportional to the number of cells the primitive intersects. In this heuristic, if we assume that there are no shared primitives among screen regions, then the sum of the weights of individual mesh cells forming a region gives the number of primitives in that region. Shared primitives may still cause some errors, but such errors are much less than counting such primitives multiple times while adding mesh-cell weights.

Minimizing the perimeter of the resulting regions in the decomposition is expected to minimize the communication overhead due to the shared primitives. 1D decomposition, i.e., horizontal or vertical striping of the screen, suffers from unscalability. A Hilbert space-filling curve [31] is widely used for $2 \mathrm{D}$ decomposition of $2 \mathrm{D}$ nonuniform workloads. In this scheme [20], the 2D coarse mesh superimposed on the screen is traversed according to the Hilbert curve to map the 2D coarse mesh to a 1D chain of mesh cells. The load-balancing problem in this decomposition scheme then reduces to the CCP problem. Using a Hilbert curve as the space- filling curve is an implicit effort towards reducing the total perimeter, since the Hilbert curve avoids jumps during the traversal of the $2 \mathrm{D}$ coarse mesh. Note that the 1D workload array used for partitioning is a realvalued array because of the inverse-area heuristic used for computing weights of the coarse-mesh cells.

\section{Experimental results}

All algorithms were implemented in the $C$ programming language. All experiments were carried out on a workstation equipped with a $133 \mathrm{MHz}$ Power $P C$ and $64 \mathrm{MB}$ of memory. We have experimented with $P=16-$, 32-, 64-, 128-, and 256-way partitioning of each test data.

Table 2 presents the test problems we have used in our experiments. In this table, $w_{\mathrm{avg}}, w_{\min }$, and $w_{\max }$ columns display the average, minimum, and maximum task weights. The dataset for the sparse-matrix decomposition comes from matrices of linear programming test problems from the Netlib suite [11] and the IOWA Optimization Center [22]. The sparsity patterns of these matrices are obtained by multiplying the respective rectangular constraint matrices with their transposes. Note that the number of tasks in the sparse-matrix $(\mathrm{SpM})$ dataset also refers to the number of rows and columns of the respective matrix. The dataset for imagespace decomposition comes from sort-first parallel DVR of curvilinear grids blunt-fin and post representing the results of computational fluid dynamic simulations, which are commonly used in volume rendering. The raw grids consist of hexahedral elements and are converted into an unstructured tetrahedral data format by dividing each hexahedron into five tetrahedrons. Triangular faces of tetrahedrons constitute the primitives mentioned in Section 5.2. Three distinct 1D workload arrays are constructed both for blunt-fin and post as described in Section 5.2 for coarse meshes of resolutions $256 \times 256,512 \times 512$, and $1024 \times 1024$ superimposed on a screen of resolution $1024 \times 1024$. The properties of these six workload arrays are displayed in Table 2. The number of tasks is much less than coarse-mesh resolution because of the zero-weight tasks, which can be compressed to retain only nonzeroweight tasks.

The following abbreviations are used for the CCP algorithms: $H 1$ and $H 2$ refer to Miguet and Pierson's [24] heuristics, and $R B$ refers to the recursive-bisection heuristic, all described in Section 3.1. DP refers to the $O((N-P) P)$-time dynamic-programming algorithm in Fig. 1. MS refers to Manne and Sørevik's iterativerefinement algorithm in Fig. 2. $\varepsilon B S$ refers to the $\varepsilon$-approximate bisection algorithm in Fig. 4. NC- and $\mathrm{NC}$ refer to the straightforward and careful implementations of Nicol's parametric-search algorithm in 
Table 2

Properties of the test set

\begin{tabular}{|c|c|c|c|c|c|c|c|c|c|c|c|c|}
\hline \multicolumn{7}{|c|}{ Sparse-matrix dataset } & \multicolumn{6}{|c|}{ Direct volume rendering (DVR) dataset } \\
\hline \multirow[t]{3}{*}{ Name } & \multirow[t]{3}{*}{ No. of tasks $N$} & \multicolumn{4}{|c|}{ Workload: No. of nonzeros } & \multirow{3}{*}{$\begin{array}{l}\text { ex. time } \\
\text { SpMxV } \\
\text { (ms) }\end{array}$} & \multirow[t]{3}{*}{ Name } & \multirow[t]{3}{*}{ No. of tasks $N$} & \multicolumn{4}{|c|}{ Workload } \\
\hline & & \multirow[t]{2}{*}{ Total $W_{\text {tot }}$} & \multicolumn{3}{|c|}{ Per row/col (task) } & & & & \multirow{2}{*}{$\begin{array}{l}\text { Total } \\
W_{\text {tot }}\end{array}$} & \multicolumn{3}{|c|}{ Per task } \\
\hline & & & $w_{\text {avg }}$ & $w_{\min }$ & $w_{\max }$ & & & & & $w_{\text {avg }}$ & $w_{\min }$ & $w_{\max }$ \\
\hline NL & 7039 & 105089 & 14.93 & 1 & 361 & 22.55 & blunt256 & 17303 & $303 \mathrm{~K}$ & 17.54 & 0.020 & 1590.97 \\
\hline cre-d & 8926 & 372266 & 41.71 & 1 & 845 & 72.20 & blunt512 & 93231 & $314 \mathrm{~K}$ & 3.36 & 0.004 & 661.50 \\
\hline CQ9 & 9278 & 221590 & 23.88 & 1 & 702 & 45.90 & blunt 1024 & 372824 & $352 \mathrm{~K}$ & 0.94 & 0.001 & 411.04 \\
\hline ken-11 & 14694 & 82454 & 5.61 & 2 & 243 & 19.65 & post256 & 19653 & $495 \mathrm{~K}$ & 25.19 & 0.077 & 3245.50 \\
\hline $\bmod 2$ & 34774 & 604910 & 17.40 & 1 & 941 & 124.05 & post512 & 134950 & $569 \mathrm{~K}$ & 4.22 & 0.015 & 1092.00 \\
\hline world & 34506 & 582064 & 16.87 & 1 & 972 & 119.45 & post 1024 & 539994 & $802 \mathrm{~K}$ & 1.49 & 0.004 & 1546.78 \\
\hline
\end{tabular}

Figs. 5(a) and (b), respectively. Abbreviations ending with "+" are used to represent our improved versions of these algorithms. That is, $\mathrm{DP}+,{ }_{\varepsilon} \mathrm{BS}+, \mathrm{NC}+$ refer to our algorithms given in Figs. 6, 8, and 10, respectively, and MS + refers to the algorithm described in Section 4.3.1. BID refers to our bidding algorithm given in Fig. 7. EBS refers to our exact bisection algorithm given in Fig. 9. Both $\varepsilon \mathrm{BS}$ and $\varepsilon \mathrm{BS}+$ algorithms are effectively used as exact algorithms for the SpM dataset with $\varepsilon=1$ for integer-valued workload arrays in the $\mathrm{SpM}$ dataset. However, these two algorithms were not tested on the DVR dataset, since they remain approximation algorithms due to the real-valued task weights in DVR.

Table 3 compares load-balancing quality of heuristics and exact algorithms. In this table, percent loadimbalance values are computed as $100 \times\left(B-B^{*}\right) / B^{*}$, where $B$ denotes the bottleneck value of the respective partition and $B^{*}=W_{\text {tot }} / P$ denotes ideal bottleneck value. OPT values refer to load-imbalance values of optimal partitions produced by exact algorithms. Table 3 clearly shows that considerably better partitions are obtained in both datasets by using exact algorithms instead of heuristics. The quality gap between exact algorithms and heuristics increases with increasing $P$. The only exceptions to these observations are 256way partitioning of blunt 256 and post 256 , for which the $R B$ heuristic finds optimal solutions.

Table 4 compares the performances of the static separator-index bounding schemes discussed in Section 4.1. The values displayed in this table are the sums of the sizes of the separator-index ranges normalized with respect to $N$, i.e., $\sum_{p=1}^{P-1} \Delta S_{p} / N$, where $\Delta S_{p}=$ $S H_{p}-S L_{p}+1$. For each CCP instance, $(N-P) P$ represents the size of the search space for the separator indices and the total number of table entries referenced and computed by the DP algorithm. The columns labeled $L 1, L 3$, and $C 5$ display total range sizes obtained according to Lemma 4.1, Lemma 4.3 and Corollary 4.5, respectively. As seen in Table 4, proposed practical scheme $C 5$ achieves substantially better separator-index bounds than $L 1$, despite its inferior worst-case behavior (see Corollaries 4.2 and 4.6). Comparison of columns L3 and $C 5$ shows the substantial benefit of performing leftto-right and right-to-left probes with $B^{*}$ according to Lemma 4.4. Comparison of $(N-P) P$ and the $C 5$ column reveals the effectiveness of the proposed separator-index bounding scheme in restricting the search space for separator indices in both $\mathrm{SpM}$ and DVR datasets. As expected, the performance of index bounding decreases with increasing $P$ because of decreasing $N / P$ values. The numbers for the DVR dataset are better than those for the SpM dataset because of the larger $N / P$ values. In Table 4 , values less than 1 indicate that the index bounding scheme achieves nonoverlapping index ranges. As seen in the table, scheme $C 5$ reduces the total separator-index range sizes below $N$ for each CCP instance with $P \leqslant 64$ in both the SpM and DVR datasets. These results show that the proposed DP + algorithm becomes a linear-time algorithm in practice.

The efficiency of the parametric-search algorithms depends on two factors: the number of probes and the cost of each probe. The dynamic index bounding schemes proposed for parametric-search algorithms reduce the cost of an individual probe. Table 5 illustrates how the proposed parametric-search algorithms reduce the number of probes. To compare performances of EBS and $\varepsilon \mathrm{BS}+$ on the DVR dataset, we forced the $\varepsilon \mathrm{BS}+$ algorithm to find optimal partitions by running it with $\varepsilon=w_{\min }$ and then improving the resulting partition using the BID algorithm. Column $\varepsilon$ BS $+\&$ BID refers to this scheme, and values after "+" denote the number of additional bottleneck values tested by the BID algorithm. As seen in Table 5, exactly one final bottleneck-value test was needed by BID to reach an optimal partition in each CCP instance.

In Table 5, comparison of the NC- and NC columns shows that dynamic bottleneck-value bounding drastically decreases the number of probes in Nicol's algorithm. Comparison of the $\varepsilon \mathrm{BS}$ and $\varepsilon \mathrm{BS}+$ columns in the SpM dataset shows that using $B_{R B}$ instead of $W_{\text {tot }}$ 
Table 3

Percent load imbalance values

\begin{tabular}{|c|c|c|c|c|c|c|c|c|c|c|c|}
\hline \multicolumn{6}{|c|}{ Sparse-matrix dataset } & \multicolumn{6}{|c|}{ DVR dataset } \\
\hline \multicolumn{2}{|c|}{ CCP instance } & \multicolumn{3}{|c|}{ Heuristics } & \multirow[t]{2}{*}{ OPT } & \multicolumn{2}{|c|}{ CCP instance } & \multicolumn{3}{|c|}{ Heuristics } & \multirow[t]{2}{*}{ OPT } \\
\hline Name & $P$ & $H 1$ & $H 2$ & $R B$ & & Name & $P$ & $H 1$ & $H 2$ & $R B$ & \\
\hline \multirow[t]{5}{*}{ NL } & 16 & 2.60 & 2.44 & 1.20 & 0.35 & \multirow[t]{5}{*}{ blunt 256} & 16 & 4.60 & 1.20 & 0.49 & 0.34 \\
\hline & 32 & 5.02 & 5.75 & 3.44 & 0.95 & & 32 & 6.93 & 2.61 & 1.94 & 1.12 \\
\hline & 64 & 8.95 & 9.01 & 5.60 & 2.37 & & 64 & 14.52 & 9.44 & 9.44 & 2.31 \\
\hline & 128 & 33.13 & 27.16 & 22.78 & 4.99 & & 128 & 38.25 & 24.39 & 16.67 & 4.82 \\
\hline & 256 & 69.55 & 69.55 & 60.78 & 14.25 & & 256 & 96.72 & 37.03 & 34.21 & 34.21 \\
\hline \multirow[t]{5}{*}{ cre-d } & 16 & 2.27 & 0.98 & 0.53 & 0.45 & \multirow[t]{5}{*}{ blunt512 } & 16 & 0.95 & 0.98 & 0.98 & 0.16 \\
\hline & 32 & 4.19 & 4.42 & 3.74 & 1.03 & & 32 & 1.38 & 1.38 & 1.18 & 0.33 \\
\hline & 64 & 7.12 & 4.92 & 4.34 & 1.73 & & 64 & 2.87 & 2.69 & 1.66 & 0.53 \\
\hline & 128 & 25.57 & 18.73 & 16.70 & 2.88 & & 128 & 5.62 & 8.45 & 4.62 & 0.97 \\
\hline & 256 & 37.54 & 26.81 & 35.20 & 10.95 & & 256 & 14.18 & 14.33 & 9.34 & 2.28 \\
\hline \multirow[t]{5}{*}{ CQ9 } & 16 & 1.85 & 1.85 & 0.58 & 0.58 & \multirow[t]{5}{*}{ blunt 1024} & 16 & 0.94 & 0.57 & 0.95 & 0.10 \\
\hline & 32 & 5.65 & 2.88 & 2.24 & 0.90 & & 32 & 1.89 & 1.21 & 0.97 & 0.14 \\
\hline & 64 & 13.25 & 11.49 & 7.64 & 1.43 & & 64 & 4.99 & 2.16 & 1.44 & 0.26 \\
\hline & 128 & 33.96 & 32.22 & 22.34 & 3.51 & & 128 & 10.06 & 4.25 & 2.47 & 0.57 \\
\hline & 256 & 58.62 & 58.62 & 58.62 & 14.72 & & 256 & 19.65 & 9.68 & 9.68 & 0.98 \\
\hline \multirow[t]{5}{*}{ ken-11 } & 16 & 3.74 & 2.01 & 0.98 & 0.21 & \multirow[t]{5}{*}{ post256 } & 16 & 1.10 & 1.43 & 0.76 & 0.56 \\
\hline & 32 & 3.74 & 4.67 & 3.74 & 1.18 & & 32 & 3.23 & 3.98 & 3.23 & 1.11 \\
\hline & 64 & 13.17 & 13.17 & 13.17 & 1.29 & & 64 & 17.28 & 11.04 & 10.90 & 3.10 \\
\hline & 128 & 13.17 & 16.89 & 13.17 & 6.80 & & 128 & 45.35 & 29.09 & 29.09 & 8.29 \\
\hline & 256 & 50.99 & 50.99 & 50.99 & 7.11 & & 256 & 67.86 & 67.86 & 67.86 & 67.86 \\
\hline \multirow[t]{5}{*}{$\bmod 2$} & 16 & 0.06 & 0.06 & 0.06 & 0.03 & \multirow[t]{5}{*}{ post512 } & 16 & 0.49 & 1.25 & 0.33 & 0.33 \\
\hline & 32 & 0.19 & 0.19 & 0.19 & 0.07 & & 32 & 0.94 & 1.61 & 0.90 & 0.58 \\
\hline & 64 & 7.42 & 2.72 & 2.18 & 0.18 & & 64 & 4.85 & 5.33 & 4.85 & 0.94 \\
\hline & 128 & 16.15 & 6.29 & 2.46 & 0.41 & & 128 & 18.03 & 14.55 & 10.15 & 1.72 \\
\hline & 256 & 19.47 & 19.47 & 18.92 & 1.23 & & 256 & 30.03 & 25.29 & 25.29 & 3.73 \\
\hline \multirow[t]{5}{*}{ world } & 16 & 0.27 & 0.18 & 0.09 & 0.04 & \multirow[t]{5}{*}{ post1024 } & 16 & 0.70 & 0.70 & 0.53 & 0.20 \\
\hline & 32 & 1.03 & 0.37 & 0.27 & 0.08 & & 32 & 1.49 & 1.49 & 1.41 & 0.54 \\
\hline & 64 & 4.73 & 4.73 & 4.73 & 0.28 & & 64 & 2.85 & 2.85 & 1.49 & 0.91 \\
\hline & 128 & 6.37 & 6.37 & 6.37 & 0.76 & & 128 & 13.15 & 11.79 & 9.10 & 1.11 \\
\hline & 256 & 27.99 & 27.41 & 27.41 & 1.11 & & 256 & 40.50 & 13.37 & 14.19 & 2.54 \\
\hline \multicolumn{12}{|c|}{ Averages over $P$} \\
\hline & 16 & 1.76 & 1.15 & 0.74 & 0.36 & & 16 & 1.44 & 1.01 & 1.04 & 0.28 \\
\hline & 32 & 3.99 & 3.39 & 2.88 & 0.76 & & 32 & 2.64 & 2.05 & 1.61 & 0.64 \\
\hline & 64 & 9.01 & 6.78 & 5.69 & 1.43 & & 64 & 7.89 & 5.58 & 4.96 & 1.31 \\
\hline & 128 & 19.97 & 17.66 & 14.09 & 3.36 & & 128 & 21.74 & 15.42 & 12.02 & 2.91 \\
\hline & 256 & 43.89 & 38.91 & 36.04 & 9.18 & & 256 & 44.82 & 27.93 & 26.76 & 18.60 \\
\hline
\end{tabular}

for the upper bound on the bottleneck values considerably reduces the number of probes. Comparison of the ${ }_{\varepsilon} \mathrm{BS}+$ and EBS columns reveals two different behaviors on SpM and DVR datasets. The discretized dynamic bottleneck-value bounding used in EBS produces only minor improvement on the SpM dataset because of the already discrete nature of integer-valued workload arrays. However, the effect of discretized dynamic bottleneck-value bounding is significant on the realvalued workload arrays of the DVR dataset.

Tables 6 and 7 display the execution times of CCP algorithms on the SpM and DVR datasets, respectively.
In Table 6, the execution times are given as percents of single $\mathrm{SpMxV}$ times. For the DVR dataset, actual execution times (in msecs) are split into prefix-sum times and partitioning times. In Tables 6 and 7, execution times of existing algorithms and their improved versions are listed in the same order under each respective classification so that improvements can be seen easily. In both tables, BID is listed separately since it is a new algorithm. Results of both $\varepsilon \mathrm{BS}$ and $\varepsilon \mathrm{BS}+$ are listed in Table 6, since they are used as exact algorithms on SpM dataset with $\varepsilon=1$. Since neither $\varepsilon$ BS nor $\varepsilon \mathrm{BS}+$ can be used as an exact algorithm on the DVR dataset, EBS, as 
Table 4

Sizes of separator-index ranges normalized with respect to $N$

\begin{tabular}{|c|c|c|c|c|c|c|c|c|c|c|c|}
\hline \multicolumn{6}{|c|}{ Sparse-matrix dataset } & \multicolumn{6}{|c|}{ DVR dataset } \\
\hline \multicolumn{2}{|c|}{ CCP instance } & \multirow{2}{*}{$(N-P) P$} & \multirow[t]{2}{*}{$L 1$} & \multirow[t]{2}{*}{$L 3$} & \multirow[t]{2}{*}{$C 5$} & \multicolumn{2}{|c|}{$\mathrm{CCP}$ instance } & \multirow{2}{*}{$(N-P) P$} & \multirow[t]{2}{*}{$L 1$} & \multirow[t]{2}{*}{$L 3$} & \multirow[t]{2}{*}{$C 5$} \\
\hline Name & $P$ & & & & & Name & $P$ & & & & \\
\hline \multirow[t]{5}{*}{ NL } & 16 & 15.97 & 0.32 & 0.13 & 0.036 & \multirow[t]{5}{*}{ blunt256 } & 16 & 15.99 & 0.38 & 0.07 & 0.003 \\
\hline & 32 & 31.86 & 1.23 & 0.58 & 0.198 & & 32 & 31.94 & 1.22 & 0.30 & 0.144 \\
\hline & 64 & 63.43 & 4.97 & 2.24 & 0.964 & & 64 & 63.77 & 5.10 & 3.81 & 0.791 \\
\hline & 128 & 125.69 & 19.81 & 19.60 & 4.305 & & 128 & 127.06 & 21.66 & 12.88 & 2.784 \\
\hline & 256 & 246.73 & 77.96 & 82.83 & 22.942 & & 256 & 252.23 & 101.68 & 50.48 & 10.098 \\
\hline \multirow[t]{5}{*}{ cre-d } & 16 & 15.97 & 0.16 & 0.04 & 0.019 & \multirow[t]{5}{*}{ blunt512 } & 16 & 16.00 & 0.09 & 0.17 & 0.006 \\
\hline & 32 & 31.89 & 0.76 & 0.90 & 0.137 & & 32 & 31.99 & 0.35 & 0.26 & 0.043 \\
\hline & 64 & 63.55 & 2.89 & 1.85 & 0.610 & & 64 & 63.96 & 1.64 & 0.69 & 0.144 \\
\hline & 128 & 126.18 & 11.59 & 15.12 & 2.833 & & 128 & 127.83 & 6.65 & 4.59 & 0.571 \\
\hline & 256 & 248.69 & 46.66 & 57.54 & 16.510 & & 256 & 255.30 & 28.05 & 16.71 & 2.262 \\
\hline \multirow[t]{5}{*}{ CQ9 } & 16 & 15.97 & 0.30 & 0.03 & 0.023 & \multirow[t]{5}{*}{ blunt1024 } & 16 & 16.00 & 0.05 & 0.09 & 0.010 \\
\hline & 32 & 31.89 & 1.21 & 0.34 & 0.187 & & 32 & 32.00 & 0.18 & 0.18 & 0.018 \\
\hline & 64 & 63.57 & 4.84 & 2.96 & 0.737 & & 64 & 63.99 & 0.77 & 0.74 & 0.068 \\
\hline & 128 & 126.25 & 19.20 & 18.66 & 3.121 & & 128 & 127.96 & 3.43 & 2.53 & 0.300 \\
\hline & 256 & 248.96 & 74.84 & 86.80 & 23.432 & & 256 & 255.82 & 13.92 & 20.36 & 1.648 \\
\hline \multirow[t]{5}{*}{ ken-11 } & 16 & 15.98 & 0.28 & 0.11 & 0.024 & \multirow[t]{5}{*}{ post 256} & 16 & 15.99 & 0.35 & 0.04 & 0.021 \\
\hline & 32 & 31.93 & 1.10 & 1.13 & 0.262 & & 32 & 31.95 & 1.50 & 0.52 & 0.162 \\
\hline & 64 & 63.73 & 4.42 & 7.34 & 0.655 & & 64 & 63.79 & 6.12 & 4.26 & 0.932 \\
\hline & 128 & 126.89 & 17.60 & 14.56 & 4.865 & & 128 & 127.17 & 26.48 & 23.68 & 4.279 \\
\hline & 256 & 251.56 & 69.18 & 85.44 & 13.076 & & 256 & 252.68 & 124.76 & 90.48 & 16.485 \\
\hline \multirow[t]{5}{*}{$\bmod 2$} & 16 & 15.99 & 0.16 & 0.00 & 0.002 & \multirow[t]{5}{*}{ post512 } & 16 & 16.00 & 0.13 & 0.02 & 0.003 \\
\hline & 32 & 31.97 & 0.55 & 0.04 & 0.013 & & 32 & 31.99 & 0.56 & 0.14 & 0.063 \\
\hline & 64 & 63.88 & 2.14 & 1.22 & 0.109 & & 64 & 63.97 & 2.33 & 2.41 & 0.413 \\
\hline & 128 & 127.53 & 8.62 & 2.59 & 0.411 & & 128 & 127.88 & 9.33 & 10.15 & 1.714 \\
\hline & 256 & 254.12 & 34.49 & 39.02 & 2.938 & & 256 & 255.52 & 37.08 & 46.28 & 6.515 \\
\hline \multirow[t]{5}{*}{ world } & 16 & 15.99 & 0.15 & 0.01 & 0.004 & \multirow[t]{5}{*}{ post1024 } & 16 & 16.00 & 0.17 & 0.07 & 0.020 \\
\hline & 32 & 31.97 & 0.59 & 0.06 & 0.020 & & 32 & 32.00 & 0.54 & 0.27 & 0.087 \\
\hline & 64 & 63.88 & 2.30 & 2.81 & 0.158 & & 64 & 63.99 & 2.19 & 0.61 & 0.210 \\
\hline & 128 & 127.53 & 9.23 & 7.19 & 0.850 & & 128 & 127.97 & 8.77 & 9.48 & 0.918 \\
\hline & 256 & 254.11 & 36.97 & 53.15 & 2.635 & & 256 & 255.88 & 34.97 & 27.28 & 4.479 \\
\hline Average & & & & & & & & & & & \\
\hline & 16 & 15.97 & 0.21 & 0.06 & 0.018 & & 16 & 16.00 & 0.20 & 0.08 & 0.011 \\
\hline & 32 & 31.88 & 0.86 & 0.68 & 0.136 & & 32 & 31.98 & 0.73 & 0.28 & 0.096 \\
\hline & 64 & 63.52 & 3.43 & 2.63 & 0.516 & & 64 & 63.91 & 3.03 & 2.09 & 0.426 \\
\hline & 128 & 126.07 & 13.68 & 12.74 & 2.731 & & 128 & 127.65 & 12.72 & 10.55 & 1.428 \\
\hline & 256 & 248.26 & 54.28 & 57.55 & 14.089 & & 256 & 254.57 & 56.74 & 41.93 & 6.915 \\
\hline
\end{tabular}

$L 1, L 3$ and $C 5$ denote separator-index ranges obtained according to results of Lemmas 4.1, 4.3 and Corollary 4.5.

an exact algorithm for general workload arrays, is listed separately in Table 7.

As seen in Tables 6 and 7, the $R B$ heuristic is faster than both $H 1$ and $H 2$ for both SpM and DVR datasets. As also seen in Table 3, $R B$ finds better partitions than both $H 1$ and $H 2$ for both datasets. These results reveal $R B$ 's superiority to $H 1$ and $H 2$.

In Tables 6 and 7, relative performance comparison of existing exact CCP algorithms shows that $\mathrm{NC}$ is two orders of magnitude faster than both DP and MS for both $\mathrm{SpM}$ and DVR datasets, and $\varepsilon \mathrm{BS}$ is considerably faster than NC for the SpM dataset. These results show that among existing algorithms the parametric-search approach leads to faster algorithms than both the dynamic-programming and the iterative-improvement approaches.

Tables 6 and 7 show that our improved algorithms are significantly faster than the respective existing algorithms. In the dynamic-programming approach, DP + is two-to-three orders of magnitude faster than DP so that 
Table 5

Number of probes performed by parametric search algorithms

\begin{tabular}{|c|c|c|c|c|c|c|c|c|c|c|c|c|c|c|}
\hline \multicolumn{8}{|c|}{ Sparse-matrix dataset } & \multicolumn{7}{|c|}{ DVR dataset } \\
\hline \multicolumn{2}{|c|}{$\mathrm{CCP}$ instance } & \multicolumn{6}{|c|}{ Parametric search algorithms } & \multicolumn{2}{|c|}{$\mathrm{CCP}$ instance } & \multicolumn{5}{|c|}{ Parametric search algorithms } \\
\hline Name & $P$ & $\mathrm{NC}-$ & $\mathrm{NC}$ & $\mathrm{NC}+$ & $\varepsilon \mathrm{BS}$ & $\varepsilon \mathrm{BS}+$ & EBS & Name & $P$ & $\mathrm{NC}-$ & $N C$ & $\mathrm{NC}+$ & $\varepsilon \mathrm{BS}+\& B I D$ & EBS \\
\hline \multirow[t]{5}{*}{ NL } & 16 & 177 & 21 & 7 & 17 & 6 & 7 & \multirow[t]{5}{*}{ blunt 256} & 16 & 202 & 18 & 6 & $16+1$ & 9 \\
\hline & 32 & 352 & 19 & 7 & 17 & 7 & 7 & & 32 & 407 & 19 & 7 & $19+1$ & 10 \\
\hline & 64 & 720 & 27 & 5 & 16 & 7 & 6 & & 64 & 835 & 18 & 10 & $21+1$ & 12 \\
\hline & 128 & 1450 & 40 & 14 & 17 & 8 & 8 & & 128 & 1686 & 25 & 15 & $22+1$ & 13 \\
\hline & 256 & 2824 & 51 & 14 & 16 & 8 & 8 & & 256 & 3556 & 203 & 62 & $23+1$ & 11 \\
\hline \multirow[t]{5}{*}{ cre-d } & 16 & 190 & 20 & 6 & 19 & 7 & 5 & \multirow[t]{5}{*}{ blunt512 } & 16 & 245 & 20 & 8 & $17+1$ & 9 \\
\hline & 32 & 393 & 25 & 11 & 19 & 9 & 8 & & 32 & 502 & 24 & 13 & $18+1$ & 10 \\
\hline & 64 & 790 & 24 & 10 & 19 & 8 & 6 & & 64 & 1003 & 23 & 11 & $18+1$ & 12 \\
\hline & 128 & 1579 & 27 & 10 & 19 & 9 & 9 & & 128 & 2023 & 26 & 12 & $20+1$ & 13 \\
\hline & 256 & 3183 & 37 & 13 & 18 & 9 & 9 & & 256 & 4051 & 23 & 12 & $21+1$ & 13 \\
\hline \multirow[t]{5}{*}{ CQ9 } & 16 & 182 & 19 & 3 & 18 & 6 & 5 & \multirow[t]{5}{*}{ blunt 1024} & 16 & 271 & 21 & 10 & $16+1$ & 13 \\
\hline & 32 & 373 & 22 & 8 & 18 & 8 & 7 & & 32 & 557 & 24 & 12 & $18+1$ & 12 \\
\hline & 64 & 740 & 29 & 12 & 18 & 8 & 8 & & 64 & 1127 & 21 & 11 & $18+1$ & 12 \\
\hline & 128 & 1492 & 40 & 12 & 17 & 8 & 8 & & 128 & 2276 & 28 & 13 & $19+1$ & 13 \\
\hline & 256 & 2971 & 50 & 14 & 18 & 9 & 9 & & 256 & 4564 & 27 & 16 & $21+1$ & 15 \\
\hline \multirow[t]{5}{*}{ ken-11 } & 16 & 185 & 21 & 6 & 17 & 6 & 6 & \multirow[t]{5}{*}{ post 256} & 16 & 194 & 22 & 9 & $18+1$ & 7 \\
\hline & 32 & 364 & 20 & 6 & 17 & 6 & 6 & & 32 & 409 & 19 & 8 & $20+1$ & 12 \\
\hline & 64 & 721 & 48 & 9 & 17 & 7 & 7 & & 64 & 823 & 17 & 11 & $22+1$ & 12 \\
\hline & 128 & 1402 & 61 & 8 & 16 & 7 & 7 & & 128 & 1653 & 19 & 13 & $22+1$ & 14 \\
\hline & 256 & 2783 & 96 & 7 & 17 & 8 & 8 & & 256 & 3345 & 191 & 102 & $23+1$ & 13 \\
\hline \multirow[t]{5}{*}{$\bmod 2$} & 16 & 210 & 20 & 6 & 20 & 5 & 4 & \multirow[t]{5}{*}{ post512 } & 16 & 235 & 24 & 9 & $16+1$ & 10 \\
\hline & 32 & 432 & 26 & 8 & 19 & 5 & 5 & & 32 & 485 & 23 & 12 & $18+1$ & 11 \\
\hline & 64 & 867 & 24 & 6 & 19 & 8 & 8 & & 64 & 975 & 22 & 13 & $20+1$ & 14 \\
\hline & 128 & 1727 & 38 & 7 & 20 & 7 & 7 & & 128 & 1975 & 25 & 17 & $21+1$ & 14 \\
\hline & 256 & 3444 & 47 & 9 & 20 & 9 & 9 & & 256 & 3947 & 29 & 20 & $22+1$ & 15 \\
\hline \multirow[t]{5}{*}{ world } & 16 & 211 & 22 & 6 & 19 & 5 & 4 & \multirow[t]{5}{*}{ post1024 } & 16 & 261 & 27 & 10 & $17+1$ & 13 \\
\hline & 32 & 424 & 26 & 5 & 19 & 6 & 6 & & 32 & 538 & 23 & 13 & $19+1$ & 14 \\
\hline & 64 & 865 & 30 & 12 & 19 & 9 & 9 & & 64 & 1090 & 22 & 12 & $17+1$ & 14 \\
\hline & 128 & 1730 & 44 & 10 & 19 & 8 & 8 & & 128 & 2201 & 25 & 15 & $21+1$ & 16 \\
\hline & 256 & 3441 & 44 & 11 & 19 & 10 & 10 & & 256 & 4428 & 28 & 16 & $22+1$ & 16 \\
\hline \multicolumn{15}{|c|}{ Averages over $P$} \\
\hline & 16 & 193 & 20.5 & 5.7 & 18.5 & 5.8 & 5.2 & & 16 & 235 & 22.0 & 8.7 & $16.7+1$ & 10.2 \\
\hline & 32 & 390 & 23.0 & 7.5 & 18.3 & 6.8 & 6.5 & & 32 & 483 & 22.0 & 10.8 & $18.7+1$ & 11.5 \\
\hline & 64 & 784 & 30.3 & 9.0 & 18.0 & 7.8 & 7.3 & & 64 & 976 & 20.5 & 11.3 & $19.3+1$ & 12.7 \\
\hline & 128 & 1564 & 41.7 & 10.2 & 18.0 & 7.8 & 7.8 & & 128 & 1969 & 24.7 & 14.2 & $20.8+1$ & 13.8 \\
\hline & 256 & 3108 & 54.2 & 11.3 & 18.0 & 8.8 & 8.8 & & 256 & 3982 & 83.5 & 38.0 & $22.0+1$ & 13.8 \\
\hline
\end{tabular}

$\mathrm{DP}+$ is competitive with the parametric-search algorithms. For the $\mathrm{SpM}$ dataset, $\mathrm{DP}+$ is 630 times faster than DP on average in 16-way partitioning, and this ratio decreases to $378,189,106$, and 56 with increasing number of processors. For the DVR dataset, if the initial prefix-sum is not included, DP + is 1277 , 578, 332, 159, and 71 times faster than DP for $P=16,32,64,128$, and 256 , respectively, on average. This decrease is expected because the effectiveness of separator-index bounding decreases with increasing $P$. These experimental findings agree with the variation in the effectiveness of separator-index bounding values seen in Table 4. In the iterative refinement approach, MS + is also one-to-three orders of magnitude faster than MS, where this drastic improvement simply depends on the scheme used for finding an initial leftist partition.

As Tables 6 and 7 reveal, significant improvement ratios are also obtained for the parametric search algorithms. On average, $\mathrm{NC}+$ is $4.2,3.5,3.1,3.7$, and 3.7 times faster than $\mathrm{NC}$ for $P=16,32,64,128$, and 256 , respectively, for the SpM dataset. For the DVR dataset, if the initial prefix-sum time is not included, $\mathrm{NC}+$ is $4.2,3.2,2.6,2.5$, and 2.7 times faster than $\mathrm{NC}$ for $P=16,32,64,128$, and 256 , respectively. For the 
Table 6

Partitioning times for sparse-matrix dataset as percents of $\mathrm{SpMxV}$ times

\begin{tabular}{|c|c|c|c|c|c|c|c|c|c|c|c|c|c|}
\hline \multicolumn{2}{|c|}{ CCP instance } & \multicolumn{3}{|c|}{ Heuristics } & \multicolumn{9}{|c|}{ Exact algorithms } \\
\hline \multirow[t]{2}{*}{ Name } & \multirow[t]{2}{*}{$P$} & \multirow[t]{2}{*}{$H 1$} & \multirow[t]{2}{*}{$H 2$} & \multirow[t]{2}{*}{$R B$} & \multicolumn{4}{|c|}{ Existing } & \multicolumn{5}{|c|}{ Proposed } \\
\hline & & & & & DP & MS & $\varepsilon B S$ & $\mathrm{NC}$ & $\mathrm{DP}+$ & $\mathrm{MS}+$ & $\varepsilon \mathrm{BS}+$ & $\mathrm{NC}+$ & BID \\
\hline \multirow[t]{5}{*}{ NL } & 16 & 0.09 & 0.09 & 0.09 & 93 & 119 & 0.93 & 1.20 & 0.40 & 0.44 & 0.40 & 0.40 & 0.22 \\
\hline & 32 & 0.18 & 0.18 & 0.13 & 177 & 194 & 1.77 & 2.17 & 1.73 & 1.73 & 0.80 & 0.80 & 0.44 \\
\hline & 64 & 0.35 & 0.35 & 0.31 & 367 & 307 & 3.15 & 5.85 & 5.81 & 4.12 & 1.86 & 1.77 & 1.82 \\
\hline & 128 & 0.89 & 0.84 & 0.71 & 748 & 485 & 6.30 & 17.12 & 19.87 & 26.92 & 4.26 & 7.32 & 4.21 \\
\hline & 256 & 1.51 & 1.55 & 1.37 & 1461 & 757 & 11.09 & 40.31 & 91.80 & 96.41 & 9.80 & 15.70 & 21.06 \\
\hline \multirow[t]{5}{*}{ cre-d } & 16 & 0.03 & 0.01 & 0.01 & 33 & 36 & 0.33 & 0.37 & 0.12 & 0.06 & 0.10 & 0.11 & 0.03 \\
\hline & 32 & 0.04 & 0.06 & 0.06 & 71 & 61 & 0.65 & 0.93 & 0.47 & 1.20 & 0.29 & 0.33 & 0.10 \\
\hline & 64 & 0.12 & 0.12 & 0.08 & 147 & 98 & 1.22 & 1.69 & 1.66 & 1.83 & 0.61 & 0.71 & 0.21 \\
\hline & 128 & 0.28 & 0.29 & 0.14 & 283 & 150 & 2.41 & 3.59 & 5.47 & 10.94 & 1.68 & 1.68 & 0.61 \\
\hline & 256 & 0.53 & 0.54 & 0.25 & 571 & 221 & 4.20 & 10.22 & 27.05 & 26.02 & 3.30 & 4.46 & 1.20 \\
\hline \multirow[t]{5}{*}{ CQ9 } & 16 & 0.04 & 0.04 & 0.04 & 59 & 73 & 0.48 & 0.59 & 0.20 & 0.13 & 0.15 & 0.13 & 0.17 \\
\hline & 32 & 0.09 & 0.09 & 0.07 & 127 & 120 & 0.94 & 1.31 & 0.94 & 0.72 & 0.41 & 0.41 & 0.28 \\
\hline & 64 & 0.20 & 0.17 & 0.15 & 248 & 195 & 1.85 & 3.18 & 3.01 & 4.47 & 1.00 & 1.44 & 0.68 \\
\hline & 128 & 0.44 & 0.44 & 0.31 & 485 & 303 & 3.18 & 8.52 & 9.65 & 18.82 & 2.44 & 3.05 & 2.51 \\
\hline & 256 & 0.92 & 0.92 & 0.63 & 982 & 469 & 6.51 & 20.33 & 69.56 & 72.2 & 5.32 & 7.45 & 14.92 \\
\hline \multirow[t]{5}{*}{ ken-11 } & 16 & 0.10 & 0.10 & 0.10 & 238 & 344 & 1.27 & 1.88 & 0.56 & 0.61 & 0.46 & 0.41 & 0.25 \\
\hline & 32 & 0.20 & 0.20 & 0.15 & 501 & 522 & 2.29 & 3.10 & 3.82 & 4.78 & 1.22 & 1.17 & 1.63 \\
\hline & 64 & 0.46 & 0.46 & 0.36 & 993 & 778 & 4.48 & 13.08 & 9.41 & 24.78 & 3.10 & 3.46 & 2.29 \\
\hline & 128 & 1.17 & 1.17 & 0.71 & 1876 & 1139 & 9.21 & 39.59 & 50.13 & 50.03 & 6.41 & 6.62 & 17.4 \\
\hline & 256 & 2.14 & 2.14 & 1.42 & 3827 & 1706 & 17.76 & 119.13 & 129.01 & 241.48 & 14.91 & 14.50 & 29.11 \\
\hline \multirow[t]{5}{*}{$\bmod 2$} & 16 & 0.02 & 0.02 & 0.01 & 92 & 119 & 0.27 & 0.34 & 0.07 & 0.05 & 0.06 & 0.06 & 0.03 \\
\hline & 32 & 0.04 & 0.04 & 0.02 & 188 & 192 & 0.51 & 0.78 & 0.19 & 0.18 & 0.16 & 0.15 & 0.07 \\
\hline & 64 & 0.11 & 0.10 & 0.06 & 378 & 307 & 1.04 & 1.91 & 0.86 & 2.18 & 0.51 & 0.55 & 0.23 \\
\hline & 128 & 0.24 & 0.23 & 0.11 & 751 & 482 & 2.28 & 5.92 & 2.61 & 3.72 & 1.06 & 1.23 & 0.53 \\
\hline & 256 & 0.48 & 0.48 & 0.23 & 1486 & 756 & 4.26 & 11.14 & 12.11 & 50.04 & 2.91 & 3.43 & 3.66 \\
\hline \multirow[t]{5}{*}{ world } & 16 & 0.02 & 0.02 & 0.02 & 99 & 122 & 0.29 & 0.33 & 0.08 & 0.05 & 0.07 & 0.08 & 0.03 \\
\hline & 32 & 0.04 & 0.04 & 0.04 & 198 & 196 & 0.59 & 0.88 & 0.26 & 0.21 & 0.18 & 0.19 & 0.08 \\
\hline & 64 & 0.12 & 0.11 & 0.09 & 385 & 306 & 1.16 & 1.70 & 1.09 & 4.84 & 0.64 & 0.54 & 0.35 \\
\hline & 128 & 0.24 & 0.23 & 0.19 & 769 & 496 & 2.19 & 5.32 & 4.48 & 10.15 & 1.31 & 1.23 & 1.77 \\
\hline & 256 & 0.47 & 0.47 & 0.36 & 1513 & 764 & 4.06 & 11.83 & 11.54 & 69.99 & 3.46 & 3.50 & 2.48 \\
\hline \multicolumn{14}{|c|}{ Averages over $P$} \\
\hline & 16 & 0.05 & 0.05 & 0.05 & 102 & 136 & 0.60 & 0.78 & 0.24 & 0.22 & 0.21 & 0.20 & 0.12 \\
\hline & 32 & 0.10 & 0.10 & 0.08 & 210 & 214 & 1.12 & 1.53 & 1.23 & 1.47 & 0.51 & 0.51 & 0.43 \\
\hline & 64 & 0.23 & 0.22 & 0.18 & 420 & 332 & 2.15 & 4.57 & 3.64 & 7.04 & 1.29 & 1.41 & 0.93 \\
\hline & 128 & 0.54 & 0.53 & 0.36 & 819 & 509 & 4.26 & 13.34 & 15.37 & 20.1 & 2.86 & 3.52 & 4.51 \\
\hline & 256 & 1.01 & 1.01 & 0.71 & 1640 & 779 & 7.98 & 35.49 & 56.84 & 92.69 & 6.62 & 8.17 & 12.07 \\
\hline
\end{tabular}

SpM dataset, $\varepsilon \mathrm{BS}+$ is $3.4,2.5,1.8,1.6$, and 1.3 times faster than $\varepsilon \mathrm{BS}$ for $P=16,32,64,128$, and 256 , respectively. These improvement ratios in the execution times of the parametric search algorithms are below the improvement ratios in the numbers of probes displayed in Table 5. Overhead due to the $R B$ call and initial settings of the separator indices contributes to this difference in both $\mathrm{NC}+$ and $\varepsilon \mathrm{BS}+$. Furthermore, costs of initial probes with very large bottleneck values are very cheap in $\varepsilon$ BS.

In Table 6, relative performance comparison of the proposed exact CCP algorithms shows that BID is the clear winner for small to moderate $P$ values (i.e., $P \leqslant 64$ ) in the SpM dataset. Relative performance of BID degrades with increasing $P$ so that both $\varepsilon \mathrm{BS}+$ and $\mathrm{NC}+$ begin to run faster than BID for $P \geqslant 128$ in general, where $\varepsilon \mathrm{BS}+$ becomes the winner. For the DVR dataset, $\mathrm{NC}+$ and EBS are clear winners as seen in Table 7. NC + runs slightly faster than EBS for $P \leqslant 128$, but EBS runs considerably faster than $\mathrm{NC}+$ for $P=256$. BID can compete with these two algorithms only for 16- and 32-way partitioning of grid blunt-fin (for all mesh resolutions). As seen in Table 6, BID takes less than $1 \%$ of a single SpMxV time 
Table 7

Partitioning times (in msecs) for DVR dataset

\begin{tabular}{|c|c|c|c|c|c|c|c|c|c|c|c|c|c|}
\hline \multicolumn{2}{|c|}{ CCP instance } & \multirow{3}{*}{$\begin{array}{l}\text { Prefix } \\
\text { sum }\end{array}$} & \multicolumn{3}{|c|}{ Heuristics } & \multicolumn{8}{|c|}{ Exact algorithms } \\
\hline \multirow[t]{2}{*}{ Name } & \multirow[t]{2}{*}{$P$} & & \multirow[t]{2}{*}{$H 1$} & \multirow[t]{2}{*}{$H 2$} & \multirow[t]{2}{*}{$R B$} & \multicolumn{3}{|c|}{ Existing } & \multicolumn{5}{|c|}{ Proposed } \\
\hline & & & & & & DP & MS & $\mathrm{NC}$ & $\mathrm{DP}+$ & $\mathrm{MS}+$ & $\mathrm{NC}+$ & EBS & BID \\
\hline \multirow[t]{5}{*}{ blunt 256} & 16 & & 0.02 & 0.02 & 0.03 & 68 & 49 & 0.36 & 0.21 & 0.53 & 0.10 & 0.14 & 0.03 \\
\hline & 32 & & 0.05 & 0.05 & 0.05 & 141 & 77 & 0.77 & 0.76 & 0.76 & 0.21 & 0.27 & 0.24 \\
\hline & 64 & 1.95 & 0.11 & 0.12 & 0.09 & 286 & 134 & 1.39 & 3.86 & 8.72 & 0.64 & 0.71 & 0.78 \\
\hline & 128 & & 0.24 & 0.25 & 0.20 & 581 & 206 & 3.66 & 12.37 & 29.95 & 1.78 & 1.84 & 2.33 \\
\hline & 256 & & 0.47 & 0.50 & 0.37 & 1139 & 296 & 52.53 & 42.89 & 0.78 & 13.96 & 3.11 & 56.69 \\
\hline \multirow[t]{5}{*}{ blunt512 } & 16 & & 0.03 & 0.03 & 0.03 & 356 & 200 & 0.55 & 0.25 & 0.91 & 0.14 & 0.16 & 0.09 \\
\hline & 32 & & 0.07 & 0.07 & 0.07 & 792 & 353 & 1.31 & 1.23 & 2.13 & 0.44 & 0.43 & 0.22 \\
\hline & 64 & 13.45 & 0.18 & 0.19 & 0.14 & 1688 & 593 & 2.65 & 3.68 & 9.28 & 0.94 & 1.10 & 0.45 \\
\hline & 128 & & 0.39 & 0.40 & 0.28 & 3469 & 979 & 5.84 & 15.01 & 46.72 & 2.29 & 2.63 & 1.65 \\
\hline & 256 & & 0.74 & 0.75 & 0.54 & 7040 & 1637 & 9.70 & 57.50 & 164.49 & 5.59 & 5.95 & 10.72 \\
\hline \multirow[t]{5}{*}{ blunt1024 } & 16 & & 0.03 & 0.03 & 0.03 & 1455 & 780 & 0.75 & 0.93 & 4.77 & 0.25 & 0.28 & 0.19 \\
\hline & 32 & & 0.12 & 0.11 & 0.08 & 3251 & 1432 & 1.81 & 2.02 & 8.92 & 0.60 & 0.62 & 0.31 \\
\hline & 64 & 59.12 & 0.27 & 0.27 & 0.19 & 6976 & 2353 & 3.50 & 7.35 & 22.28 & 1.27 & 1.37 & 1.39 \\
\hline & 128 & & 0.50 & 0.51 & 0.37 & 14337 & 3911 & 9.14 & 33.01 & 69.38 & 3.36 & 3.56 & 16.21 \\
\hline & 256 & & 0.97 & 1.00 & 0.68 & 29417 & 6567 & 16.59 & 180.09 & 538.10 & 8.48 & 8.98 & 16.29 \\
\hline \multirow[t]{5}{*}{ post256 } & 16 & & 0.02 & 0.03 & 0.03 & 79 & 61 & 0.46 & 0.18 & 0.13 & 0.10 & 0.11 & 0.24 \\
\hline & 32 & & 0.05 & 0.05 & 0.05 & 157 & 100 & 0.77 & 1.05 & 1.74 & 0.26 & 0.31 & 0.76 \\
\hline & 64 & 2.16 & 0.10 & 0.11 & 0.10 & 336 & 167 & 1.37 & 5.26 & 9.72 & 0.61 & 0.70 & 4.67 \\
\hline & 128 & & 0.25 & 0.26 & 0.18 & 672 & 278 & 2.90 & 22.94 & 55.10 & 1.65 & 1.77 & 23.96 \\
\hline & 256 & & 0.47 & 0.48 & 0.37 & 1371 & 338 & 45.16 & 84.78 & 0.77 & 13.04 & 3.62 & 299.32 \\
\hline \multirow[t]{5}{*}{ post512 } & 16 & & 0.02 & 0.02 & 0.03 & 612 & 454 & 0.63 & 0.20 & 0.44 & 0.14 & 0.16 & 1.21 \\
\hline & 32 & & 0.07 & 0.07 & 0.07 & 1254 & 699 & 1.30 & 2.49 & 1.95 & 0.38 & 0.42 & 3.36 \\
\hline & 64 & 20.55 & 0.18 & 0.18 & 0.13 & 2559 & 1139 & 2.58 & 16.93 & 38.73 & 0.97 & 1.07 & 8.53 \\
\hline & 128 & & 0.38 & 0.39 & 0.28 & 5221 & 1936 & 5.84 & 68.25 & 156.15 & 2.27 & 2.44 & 31.54 \\
\hline & 256 & & 0.70 & 0.73 & 0.53 & 10683 & 3354 & 12.67 & 256.48 & 726.54 & 5.44 & 5.39 & 140.88 \\
\hline \multirow[t]{5}{*}{ post1024 } & 16 & & 0.03 & 0.04 & 0.03 & 2446 & 1863 & 0.91 & 2.88 & 5.73 & 0.17 & 0.21 & 2.63 \\
\hline & 32 & & 0.09 & 0.08 & 0.08 & 5056 & 2917 & 1.61 & 13.57 & 17.37 & 0.51 & 0.58 & 12.73 \\
\hline & 64 & 69.09 & 0.25 & 0.26 & 0.17 & 10340 & 4626 & 3.56 & 35.05 & 33.25 & 1.35 & 1.47 & 32.53 \\
\hline & 128 & & 0.51 & 0.53 & 0.36 & 21102 & 7838 & 7.90 & 156.34 & 546.92 & 2.95 & 3.28 & 76.96 \\
\hline & 256 & & 0.95 & 0.98 & 0.67 & 43652 & 13770 & 16.30 & 764.59 & 1451.87 & 7.55 & 7.02 & 300.70 \\
\hline \multicolumn{14}{|c|}{ Averages normalized w.r.t. RB times (prefix-sum time not included) } \\
\hline & 16 & & 0.83 & 0.94 & 1.00 & 27863 & 18919 & 20.33 & 25.83 & 69.50 & 5.00 & 5.89 & 24.39 \\
\hline & 32 & & 1.10 & 1.06 & 1.00 & 23169 & 12156 & 18.47 & 47.37 & 72.82 & 5.83 & 6.46 & 39.02 \\
\hline & 64 & & 1.30 & 1.35 & 1.00 & 22636 & 9291 & 17.88 & 82.81 & 145.19 & 7.00 & 7.81 & 53.81 \\
\hline & 128 & & 1.35 & 1.39 & 1.00 & 22506 & 7553 & 20.46 & 168.36 & 481.19 & 8.60 & 9.31 & 86.81 \\
\hline & 256 & & 1.35 & 1.39 & 1.00 & 24731 & 6880 & 59.10 & 390.25 & 772.99 & 19.55 & 10.51 & 286.77 \\
\hline \multicolumn{14}{|c|}{ Averages normalized w.r.t. RB times (prefix-sum time included) } \\
\hline & 16 & & 1.00 & 1.00 & 1.00 & 32 & 23 & 1.08 & 1.04 & 1.09 & 1.01 & 1.02 & 1.03 \\
\hline & 32 & & 1.00 & 1.00 & 1.00 & 66 & 36 & 1.15 & 1.21 & 1.29 & 1.04 & 1.05 & 1.13 \\
\hline & 64 & & 1.00 & 1.00 & 1.00 & 135 & 58 & 1.27 & 1.97 & 2.90 & 1.11 & 1.12 & 1.55 \\
\hline & 128 & & 1.01 & 1.01 & 1.00 & 275 & 94 & 1.62 & 4.75 & 10.53 & 1.28 & 1.30 & 3.25 \\
\hline & 256 & & 1.02 & 1.02 & 1.00 & 528 & 142 & 7.98 & 14.64 & 13.71 & 2.95 & 1.55 & 26.73 \\
\hline
\end{tabular}

for $P \leqslant 64$ on average. For the DVR dataset (Table 7), the initial prefix-sum time is considerably larger than the actual partitioning time of the EBS algorithm in all CCP instances except 256-way partitioning of post 256 . As seen in Table 7, EBS is only $12 \%$ slower than the $R B$ heuristic in 64-way partitionings on average.

These experimental findings show that the proposed exact CCP algorithms should replace heuristics. 


\section{Conclusions}

We proposed runtime efficient chains-on-chains partitioning algorithms for optimal load balancing in 1-D decompositions of nonuniform computational domains. Our main idea was to run an effective heuristic, as a preprocessing step, to find a "good" upper bound on the optimal bottleneck value, and then exploit lower and upper bounds on the optimal bottleneck value to restrict the search space for separator-index values. This separator-index bounding scheme was exploited in a static manner in the dynamic-programming algorithm, drastically reducing the number of table entries computed and referenced. A dynamic separator-index bounding scheme was proposed for parametric search algorithms to narrow separator-index ranges after each probe. We enhanced the approximate bisection algorithm to be exact by updating lower and upper bounds into realizable values after each probe. We proposed a new iterative-refinement scheme, that is very fast for small-to-medium numbers of processors.

We investigated two distinct applications for experimental performance evaluation: 1D decomposition of irregularly sparse matrices for parallel matrix-vector multiplication, and decomposition for image-space parallel volume rendering. Experiments on the sparse matrix dataset showed that 64-way decompositions can be computed 100 times faster than a single sparse matrix vector multiplication, while reducing the load imbalance by a factor of four over the most effective heuristic. Experimental results on the volume rendering dataset showed that exact algorithms can produce 3.8 times better 64-way decompositions than the most effective heuristic, while being only $11 \%$ slower, on average.

\section{Availability}

The methods proposed in this work are implemented in $\mathrm{C}$ programming language and are made publicly available at http://www.cse.uiuc. edu/〜alipinar/ccp/.

\section{References}

[1] S. Anily, A. Federgruen, Structured partitioning problems, Oper. Res. 13 (1991) 130-149.

[2] C. Aykanat, F. Ozguner, Large grain parallel conjugate gradient algorithms on a hypercube multiprocessor in: Proceedings of the 1987 International Conference on Parallel Processing, Penn State University, University Park, PA, 1987, pp. 641-645.

[3] C. Aykanat, F. Ozguner, F. Ercal, P. Sadayappan, Iterative algorithms for solution of large sparse systems of linear equations on hypercubes, IEEE Trans. Comput. 37 (12) (1988) 1554-1567.

[4] S.H. Bokhari, Partitioning problems in parallel, pipelined, and distributed computing, IEEE Trans. Comput. 37 (1) (1988) 48-57.

[5] U.V. Catalyurek, C. Aykanat, Hypergraph-partitioning-based decomposition for parallel sparse-matrix vector multiplication, IEEE Trans. Parallel Distributed Systems 10 (7) (1999) 673-693.
[6] H. Choi, B. Narahari, Algorithms for mapping and partitioning chain structured parallel computations, in: Proceedings of the 1991 International Conference on Parallel Processing, Austin, TX, 1991 pp. I-625-I-628.

[7] G.N. Frederickson, Optimal algorithms for partitioning trees and locating $p$-centers in trees, Technical Report CSD-TR-1029, Purdue University (1990).

[8] G.N. Frederickson, Optimal algorithms for partitioning trees in: Proceedings of the Second ACM-SIAM Symposium Discrete Algorithms, Orlando, FL, 1991.

[9] M.R. Garey, D.S. Johnson, Computers and Intractability: A Guide to the Theory of NP-Completeness, W.H. Freeman, San Fransisco, CA, 1979.

[10] M.R. Garey, D.S. Johnson, L. Stockmeyer, Some simplified npcomplete graph problems, Theoret. Comput. Sci. 1 (1976) 237-267.

[11] D.M. Gay, Electronic mail distribution of linear programming test problems, Mathematical Programming Society COAL Newsletter.

[12] Y. Han, B. Narahari, H.-A. Choi, Mapping a chain task to chained processors, Inform. Process. Lett. 44 (1992) 141-148.

[13] P. Hansen, K.W. Lih, Improved algorithms for partitioning problems in parallel, pipelined and distributed computing, IEEE Trans. Comput. 41 (6) (1992) 769-771.

[14] B. Hendrickson, T. Kolda, Rectangular and structurally nonsymmetric sparse matrices for parallel processing, SIAM J. Sci. Comput. 21 (6) (2000) 2048-2072.

[15] M.A. Iqbal, Efficient algorithms for partitioning problems, in: Proceedings of the 1990 International Conference on Parallel Processing, Urbana, Champaign, IL, 1990, pp. III-123-127.

[16] M.A. Iqbal, Approximate algorithms for partitioning and assignment problems, Int. J. Parallel Programming 20 (5) (1991) 341-361.

[17] M.A. Iqbal, S.H. Bokhari, Efficient algorithms for a class of partitioning problems, IEEE Trans. Parallel Distributed Systems 6 (2) (1995) 170-175.

[18] M.A. Iqbal, J.H. Saltz, S.H. Bokhari, A comparative analysis of static and dynamic load balancing strategies in: Proceedings of the 1986 International Conference on Parallel Processing, Penn State Univ University Park, PA, 1986, pp. 1040-1047.

[19] V. Kumar, A. Grama, A. Gupta, G. Karypis, Introduction to Parallel Computing, Benjamin, Cummings, Redwood City, CA, 1994.

[20] H. Kutluca, T.M. Kurç, C. Aykanat, Image-space decomposition algorithms for sort-first parallel volume rendering of unstructured grids, J. Supercomput. 15 (1) (2000) 51-93.

[21] T. Lengauer, Combinatorial Algorithms for Integrated Circuit Layout, Wiley, Chichester, UK, 1990.

[22] Linear programming problems, Technical Report, IOWA Optimization Center, ftp://col.biz.uiowa.edu:pub/testprob/lp/gondzio.

[23] F. Manne, T. Sørevik, Optimal partitioning of sequences, J. Algorithms 19 (1995) 235-249.

[24] S. Miguet, J.M. Pierson, Heuristics for 1d rectilinear partitioning as a low cost and high quality answer to dynamic load balancing, Lecture Notes in Computer Science, Vol. 1225, Springer, Berlin, 1997, pp. 550-564.

[25] S. Molnar, M. Cox, D. Ellsworth, H. Fuchs, A sorting classification of parallel rendering, IEEE Comput. Grap. Appl. 14 (4) (1994) 23-32.

[26] C. Mueller, The sort-first rendering architecture for highperformance graphics, in: Proceedings of the 1995 Symposium on Interactive 3D Graphics, Monterey, CA, 1995, pp. 75-84.

[27] D.M. Nicol, Rectilinear partitioning of irregular data parallel computations, J. Parallel Distributed Computing 23 (1994) 119-134.

[28] D.M. Nicol, D.R. O'Hallaron, Improved algorithms for mapping pipelined and parallel computations, Technical Report 88-2, ICASE, 1988.

[29] D.M. Nicol, D.R. O'Hallaron, Improved algorithms for mapping pipelined and parallel computation, IEEE Trans. Comput. 40 (3) (1991) 295-306. 
[30] B. Olstad, F. Manne, Efficient partitioning of sequences, IEEE Trans. Comput. 44 (11) (1995) 1322-1326.

[31] J.R. Pilkington, S.B. Baden, Dynamic partitioning of nonuniform structured workloads with spacefilling curves, IEEE Trans. Parallel Distributed Systems 7 (3) (1996) 288-299.

[32] Y. Saad, Practical use of polynomial preconditionings for the conjugate gradient method, SIAM J. Sci. Statist. Comput. 6 (5) (1985) 865-881.

[33] M.U. Ujaldon, E.L. Zapata, B.M. Chapman, H.P. Zima, ViennaFortran/hpf extensions for sparse and irregular problems and their compilation, IEEE Trans. Parallel Distributed Systems 8 (10) (1997) 1068-1083

[34] M.U. Ujaldon, E.L. Zapata, S.D. Sharma, J. Saltz, Parallelization techniques for sparse matrix applications, J. Parallel Distributed Computing 38 (1996) 256-266.

[35] M.U. Ujaldon, E.L. Zapata, S.D. Sharma, J. Saltz, Experimental evaluation of efficient sparse matrix distributions, in: Proceedings of the ACM International Conference of Supercomputing Philadelphia, PA, (1996) pp. 78-86.

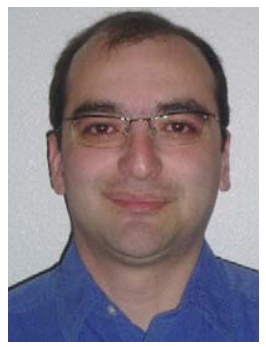

Ali Pinar's research interests are combinatorial scientific computing - the boundary between scientific computing and combinatorial algorithms - and high performance computing. He received his B.S. and M.S. degrees in Computer Science from Bilkent University, Turkey, and his Ph.D. degree in Computer Science with the option of Computational Science and Engineering from University of Illinois at Urbana-Champaign. During his $\mathrm{Ph}$.D. studies he frequently visited Sandia National Laboratories in New Mexico, which had a significant influence on his education and research. He joined the Computational
Research Division of Lawrence Berkeley National Laboratory in October, 2001, where he currently works as a research scientist.

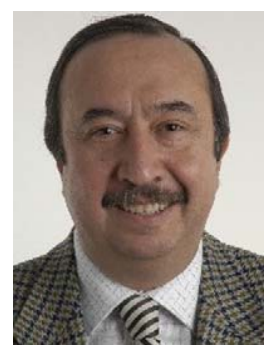

Cevdet Aykanat received the B.S. and M.S degrees from Middle East Technical University, Ankara, Turkey, both in electrical engineering, and the Ph.D. degree from Ohio State University, Columbus, in electrical and computer engineering. He was a Fulbright scholar during his Ph.D. studies. He worked at the Intel Supercomputer Systems Division, Beaverton, Oregon, as a research associate. Since 1989, he has been affiliated with the Department of Computer Engineering, Bilkent University, Ankara, Turkey, where he is currently a professor. His research interests mainly include parallel computing, parallel scientific computing and its combinatorial aspects, parallel computer graphics applications, parallel data mining, graph and hypergraph-partitioning, load balancing, neural network algorithms, high performance information retrieval and GIS systems, parallel and distributed web crawling, distributed databases, and grid computing. He is a member of the ACM and the IEEE Computer Society. He has been recently appointed as a member of IFIP Working Group 10.3 (Concurrent Systems). 